\title{
Estudo Morphologico e Estatistico da Phenacita de S. Miguel de Piracicaba
}

\author{
(10 figuras no; texto e 2 quadros)
}

\author{
R. Saldanha
}

As jazidas de S. Miguel de Piracicaba, no centro do estado de Minas Geraes ,foram estudadas por EUGENio HUSSAK (1), por indicação do engenheiro de minas L. CAVALliER DE DARBILlY, que encontrou a primeira amostra classificada por aquelle, no local da mina de ouro então alli explorada.

A phenacita, como bem descreveu Hussak no seu trabalho, occorre em veios de pegmatito com a superficie exterior completamente alterada.

E' rico o veio em mica verde, apresentando tambem grandes crystaes de amazonita e mais quartzo hyalino e quartzo enfumaçado. Raramente se encontra a turmalina (var. aphrisita) e excepcionalmente monazita, zirconio, columbita, oligisto, pyrita e granada almandina. HUSSAK cita a occurrencia de xenotima num crystal de $6 \mathrm{~m} / \mathrm{m}$ de aresta, com habitus pseudo-cubico pelo egual desenvolvimento do pinacoide basal e das faces do prisma; chama ainda a attenção para a ausencia de topazio, agua marinha e mais mineraes typicos de outros pegmatitos brasileiros, nos veios por elle estudados.

A phenacita apresenta-se em crystaes quasi sempre bj-terminados, ultrapassando em certos casos $10 \mathrm{~cm}$. na maior dimensão

(1) E. Fiussak - Uma nova jazida de phenakita no Brasil - Annaes da Escola de Minas de Ouro Preto, n. ${ }^{\circ} 15$, pg. 16 - 18, edição em 1917. Cent. f. Min., 1909, pg. 268. 
(2) Encontram-se soltos no pegmatito alterado, mas incompletos na parte que esteve anteriormente engastada.

Além de muitos exemplares opacos, de côr branco-leitosa, são numercsos os incolores e de bella transparencia, em especial os que não excedem $1 \mathrm{~cm}$. de diametro e são empregados para a lapidação como pedra semi-preciosa.

E. Hussak tendo estudado com desvelo as jazidas, não se preoccupou de modo identico com a morphologia. Os demais que, contemporanea ou posteriormente, voltaram-se para esse lado, tiveram ao dispor numero resiricto de exemplares; ficou a parte morpholcgica para ser estudada em detalhe, por quem dispusses a de farto material.

Propuzemo-nos este trabalho, graças á gentileza dos professores Theodoro VAZ (3) e ODORICo DE AlbuquERque, da Escola de Minas (Ouro Preto), aos quaes devemos o offerecimento de grande numero de amostras ao nosso Museu.

As primeiras pesquizas crystallographicas de HUSSAK foram seguidas, quasi contemporaneamente, por F. SLAviK (4) e V GOLDSCHMIDT e R. SCHRÖDER (5). Logo após apareceram os trabalhos de K. ZimánYi (6) e G. F. H. SMITH (7) e mais recentemente os de H. Butrannbach (8) e M. V Rosicky (9).

Todos esses cutores, não podendo dispor de muito material (algumas vezes 1 ou 2 amostras), apresentaram relações de fórmas encontradas que, como é natural, não podiam coincidir completamente. E' de extranhar, entretanto, que no estudo de Hussak esteja comprehendido o rhomboedro $\{321\}$ não comprovado por nenhum dos que o succederam e que não conseguimos, tampouco,

(2) Encontra-se em nosso Museu um exemplar que mede $10^{\mathrm{cm}}, 5 \times 7^{\mathrm{c} 1 \mathrm{n}} \times 3^{\mathrm{cm}, 5}$ pcrtencente á collecção Araujo Ferraz.

(3) Agradecemos tambem ao prof. THEOdoro VAz o auxilio que prestou com a remessa de trabalho já exgottado.

(4) F. SlaviK - Phenakit von Brasilien - Zeit. f. K1 ist. 50, 1912, pg. 647. Cent. f. Min. u. Geol., 1909, pgs. 264-267.

(5) V Goldschmidt u. R. Schröder - Phenakit aus Brasilien - Zeit. f. Krist, 46. 1909, pgs. 465470 .

(6) K. ZIMÁNYI - Über den Phenakit aus Brasilien - Zeit. f. Krist., 47, 1910, pgs. 97103 .

(7) G. F. H. Sмiтh - Cent. f. Min., 1910, pg. 189 e em nota (9).

(8) H. Buttgenbach - Bulletin de la classe des Sciences, Brumellas, 1924, t. X (5." série) pgs. $456-461$.

(9) M. V. Rosicky-Sur la phenacite den San Miguel de Piracicaba, Minas Geraes, (Brésil) - Bul. de la Soc. Fr. de Min., L., 1927, pags. 4.7-55. 
encontrar no grande numero de crystaes por nós medidos; ainda é digno de nota que HUSSAK não tenha verificado a existencia de fórmas muito communs na phenacita de S. Miguel de Piracicaba, como $\{22 \overline{1}\},\{210\},\{201\}$ e $\{2 \overline{1} 0\}$, presentes sempre nos exemplares dessa localidade.

No trabalho de Hussak não está indicado o criterio por elle seguido para a orientação dos crystaes. Os demais, como GoLdsCHMIDT e SCHRÖDER, ZIMÁNYI e RosickÝ, empregaram as fórmas negativas $\{12 \overline{1}\}$ e $\{31 \overline{2}\}$, de posições inversas uma da outra e que tomaram (os symbolos o demonstram) como direita e esquerda, respectivamente.

Julgamos preferivel seguir cri erio diverso, como veremos mais adiante, escolhendo entre os rhomboedros de $3 .^{\text {a }}$ ordem aquelles que possuiam maior frequencia e bom desenvolvimento. Note-se que chegamos, partindo de pontos differentes, ao mesmo resultado final.

A lista completa das fórmas encontradas, entre os varios estudiosos acima citados, para a phenacita do Brasil comprehende ao todo 26:

$$
\begin{aligned}
& \{10 \overline{1} 0\}=\{2 \overline{1} \overline{1}\},\{11 \overline{2} 0\}=\{10 \overline{1}\},\{10 \overline{1} 1\}=\{100\} \\
& \{10 \overline{1} 2\}=\{41 \overline{1},\{01 \overline{1} 1\}=\{22 \overline{1}\},\{01 \overline{1} 2\}=\{110\}, \\
& \{1 \overline{1} 23\}=\{210\},\{2 \overline{1} \overline{1} 3\}=\{201\},\{22 \overline{4} 3\}=\{31 \overline{1}\}, \\
& \{4 \overline{2} \overline{2} 3\}=\{3 \overline{1} 1\},\{21 \overline{3} 1\}=\{20 \overline{1}\},\{3 \overline{1} \overline{2} 1\}=\{2 \overline{1} 0\}, \\
& \{12 \overline{3} 2\}=\{21 \overline{1}\},\{13 \overline{2} 2\}=\{12 \overline{1}\},\{23 \overline{5} 2\}=\{31 \overline{2}\}, \\
& \{32 \overline{5} 4\}=\{41 \overline{1}\},\{5 \overline{3} \overline{3} 4=\{4 \overline{1} 1\},\{43 \overline{7} 7=\{62 \overline{1}\}, \\
& \{21 \overline{3} 4\}=\{310\},\{13 \overline{4} 4\}=\{32 \overline{1}\},\{\overline{3} 7 \overline{4} 5\}=\{25 \overline{2}\}, \\
& \{10,13, \overline{2} \overline{3}, 18\}=\{17,7, \overline{6}\},\{5,6, \overline{1} 1,11\}=\{94 \overline{2}\}, \\
& \{5,6, \overline{1} \overline{1}, 10\}=\{26,11, \overline{7}\},\{23,2, \overline{2} \overline{5}, 18\}=\{22, \overline{1}, \overline{3}\}, \\
& \{45 \overline{9} 6\}=\{19,7, \overline{8}\}
\end{aligned}
$$

Fazendo a determinação em crystaes, escolhidos entre o grande numero de que dispunhamos, chegamos não só a comprovar a occurrencia de fórmas assignaladas sómente por um dos outros autores, como a registrar 29 fórmas novas para a phenacita do Brasil, das quaes 24 são tambem novas para a especie (embora algumas prefiramos apresentar sob fórma interrogativa). 
Diante dos resultados attingidos é que julgamos opportuno publical-os, contribuindo assim para um conhecimento mais completo do mineral em questão.

Servimo-nos, para o estudo, dos pequenos exemplares porque nos permittiam medidas mais exactas e apresentavam, mesmo nos que não excediam a 3 ou $5 \mathrm{~m} / \mathrm{m}$ de tamanho, um extraordinario numero de faces. Dispondo como dissemos, de muitas dezenas de crystaes pudemos escolher aquelles cuja perfeição e limpidez se destacavam.

As formas cuja existencia verificamos são as seguintes (10):

$$
\begin{aligned}
& \mathrm{m}\{10 \overline{1}\} \text {, a }\{\overline{2} \overline{1} \overline{1}\}, \mathrm{b}\{3 \overline{1} \overline{2}\} * *(2), \mathrm{n}\{5 \overline{1} \overline{4}\} *, \text { n' }\{5 \overline{4} \overline{1}\} * \\
& \mathrm{R}\{100\} \cdot \mathrm{r}\{411\} \\
& R^{\prime}\{22 \overline{1}\}, r^{\prime}\{110\}, c\{11 \overline{1}\} * *, j\{441\} * \\
& \mathrm{p}\{210\} \text { o }\{3 \overline{1}\}, \mathrm{d}\{52 \overline{1}\} * \text {, v }\{11,2, \overline{7}\} * \\
& \text { p' }\{201\}, o^{\prime}\{3 \overline{1} 1\} \text {, d' }\{5 \overline{1} 2\} *, \text { t }\{312\} *, z\{713\} * \text { ?, } \\
& \mathrm{x}\{714\} * ?, \mathrm{y}\{534\} \text { ? } \\
& \mathrm{s}\{20 \overline{1}\}, \mathrm{s}^{\prime}\{2 \overline{1} 0\} \text {, e }\{21 \overline{1}\}, e^{\prime}\{12 \overline{1}\}, \mathrm{f}\{31 \overline{2}\} \text { f' }\{13 \overline{2}\} * \text {, } \\
& \text { g }\{43 \overline{1}\} * *, g^{\prime}\{34 \overline{1}\} * *, k\{62 \overline{1}\}, k^{\prime}\{6 \overline{1} 2\} *, h\{41 \overline{1}\} \text {, } \\
& \text { h' }\{4 \overline{1} 1\} \quad \text { l }\{310\}, q\{14 \overline{3}\} *, u\{52 \overline{4}\} * *, w\{651\} * ? \text {, } \\
& \omega\{78 \overline{3}\} * \text { ?, i }\{85 \overline{1}\} *, \alpha\{831\} *, \varepsilon\{8 \overline{1} 2\} * \text { ?, ๆ }\{2 \overline{75}\} * \text {, } \\
& \lambda\{10,7, \overline{1} \overline{1}\} * ?, \mathrm{H} \quad\{14,4, \overline{3}\} * \mathrm{H}_{1}\{14, \overline{3}, 4\} *, \mathrm{~K}\{10, \overline{2}, 3,\} * \\
& \mathrm{~K}_{1}\{16,5,3\} *
\end{aligned}
$$$$
\text { (*:*) As fórmas assim assignaladas sāo novas para phenaciala do Brasil. }
$$$$
\text { (*) As förmas assim assignaladas säo novas para especie. }
$$

$\mathrm{Na}$ relação geral é facil distinguir as 5 fórmas novas para a phenacita do Brasil e as 24 fórmas novas para o mineral como apreciar a extraordinaria multiplicidade de fórmas presentes.

De outra parte não nos foi possivel comprovar quanto aos trabalhos sobre exemplares da phenacita de São Miguel de Piracicaba a existencia das formas $\{32 \overline{1}\}$ citada por E. HussaK, $\{19,7, \overline{8}\}$

(10) Usamos aqui os indices referidos ao systema a tres eixos do trigonal. Para commodidade de consulta damos na tabella de valores angulares os correspondentes da cruz axial bravaisiana. 


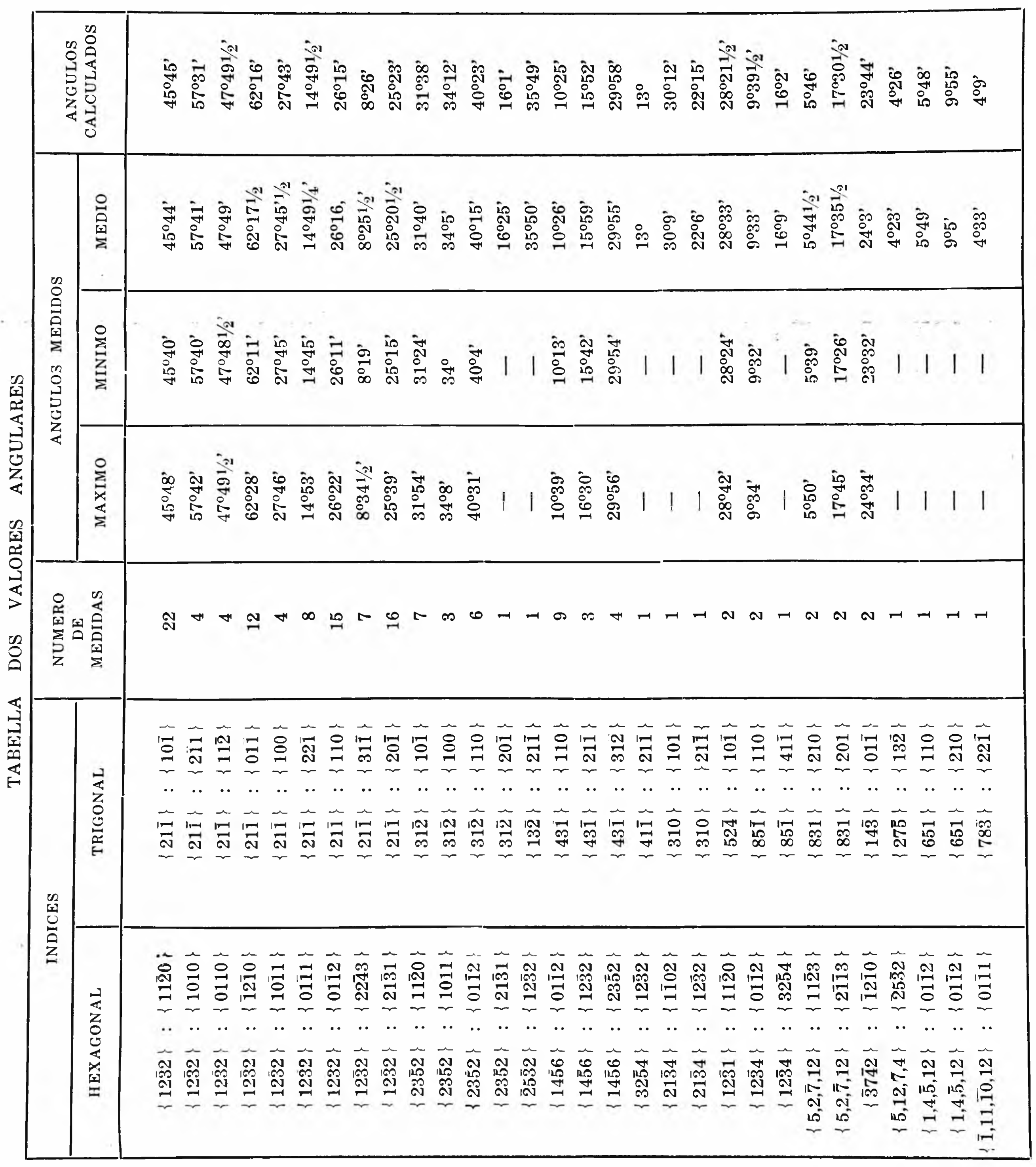




\begin{tabular}{|c|c|c|}
\hline \multicolumn{2}{|c|}{ 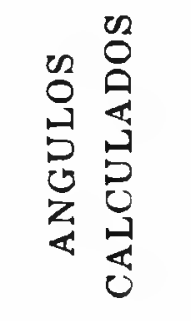 } & 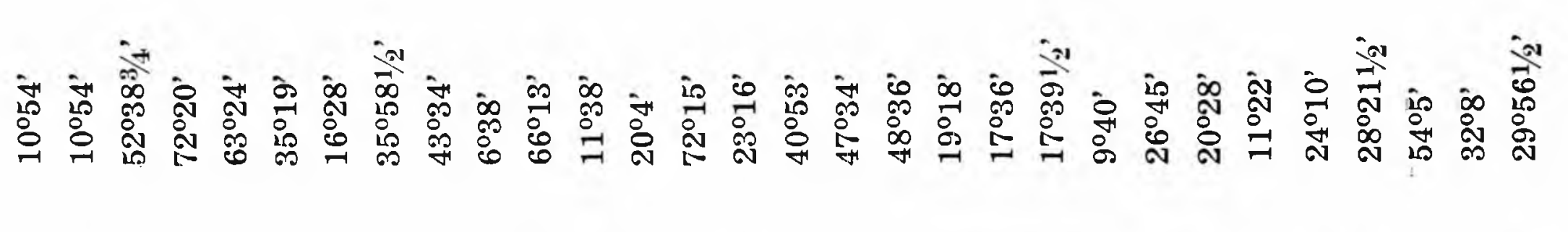 \\
\hline \multirow{3}{*}{ 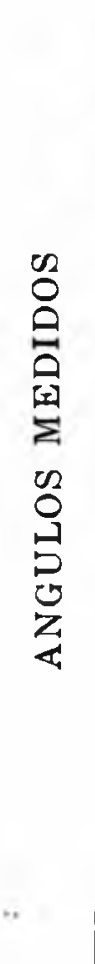 } & $\begin{array}{l}\stackrel{\circ}{9} \\
\stackrel{9}{\Sigma}\end{array}$ & 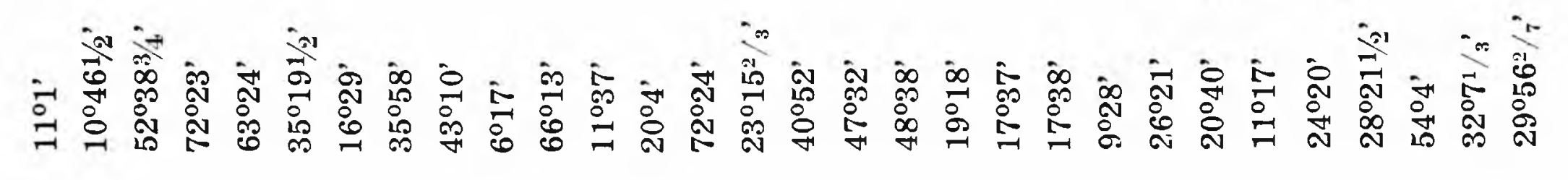 \\
\hline & 㚆 & 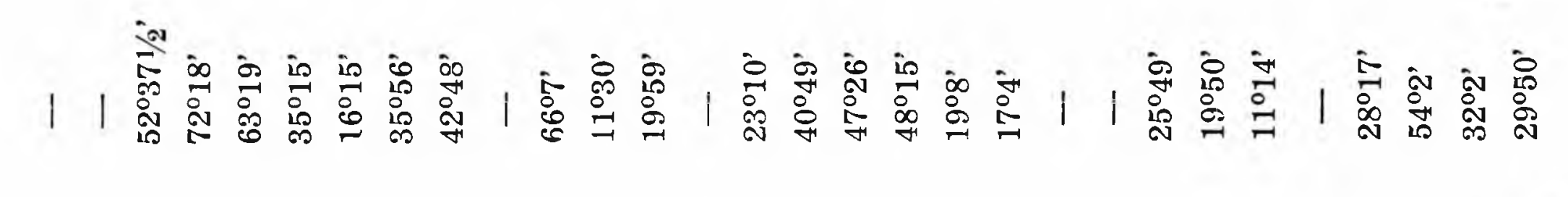 \\
\hline & 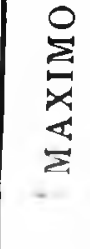 & 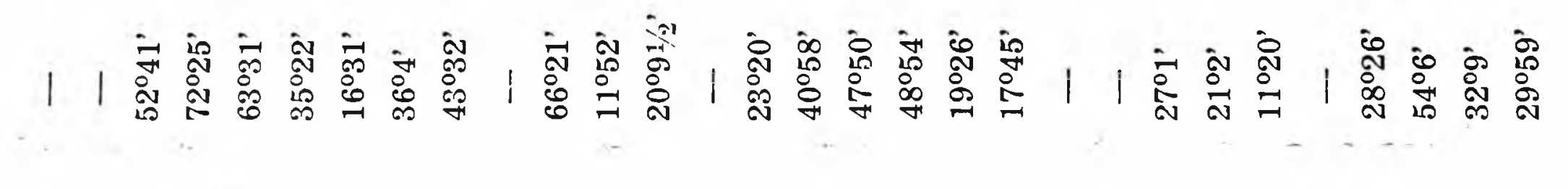 \\
\hline \multicolumn{2}{|c|}{ 蛋国最 } & $\neg \neg$ ส \\
\hline \multirow{2}{*}{ 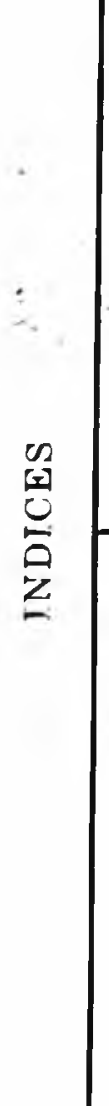 } & 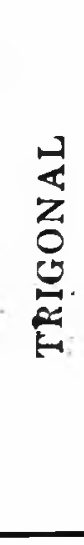 & 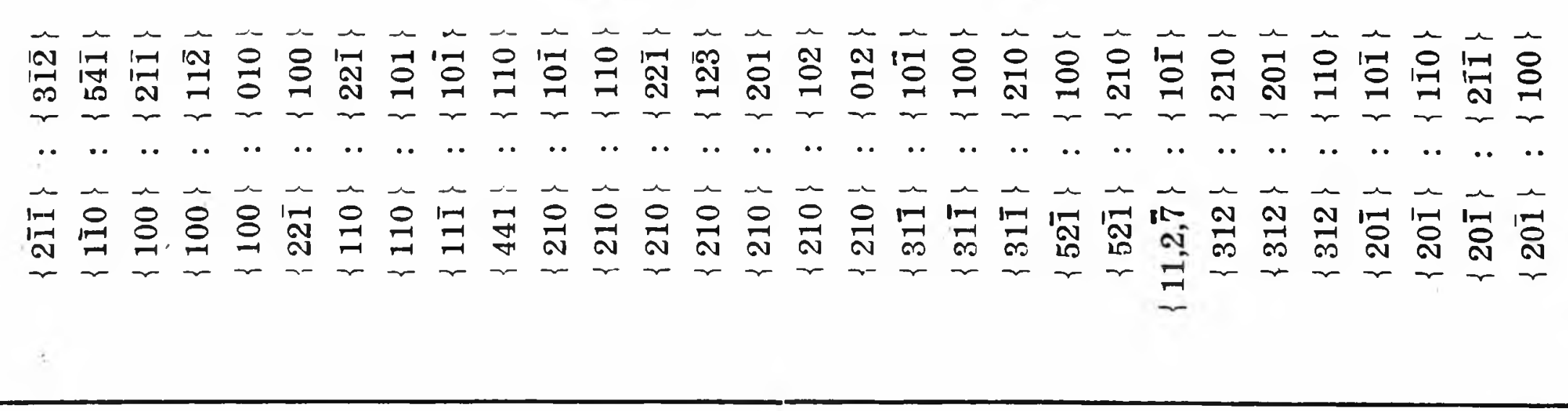 \\
\hline & 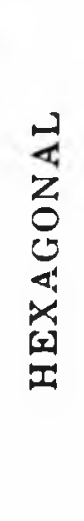 & 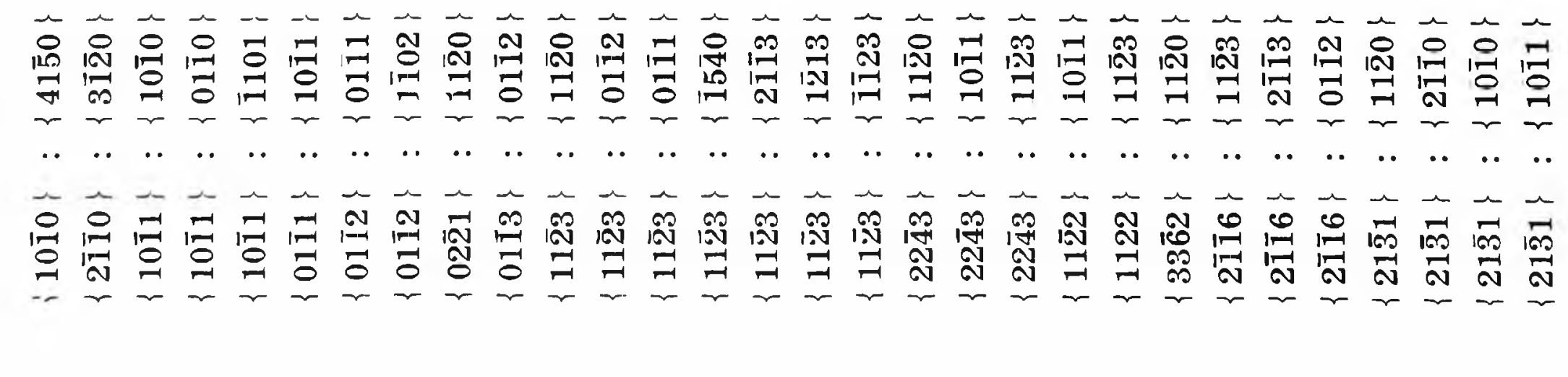 \\
\hline
\end{tabular}


por G. F. H. SMith e $\{2 \overline{5} 2\}$ por M. V RosickÝ. Ha a accrescentar nesta lista as pequenas faces encontradas por este ultimo $(22,1, \overline{3}$.) e $(26,11, \overline{7})$, a indices complicados; a segunda pode-se confundir com a face (942) assignalada por H. ButtgenbaCH, como bem accentúa RosickÝ, si admittirmos, dadas as medidas aproximativas, um erro angular superior a $2 .^{\circ}$ Como tambem conclue o mesmo autor a fórma $\mathrm{t} \mathrm{b}^{1 / 2} \mathrm{~d}^{1} \mathrm{~d} 1 / 3$ dada por $\mathrm{H}$. Buttgenbach como nova deve ser $\{31 \overline{2}\}$ que se encontra nas relações de F SLAviK, V Goldschmidt e R. SCHRöDER e V ZimánYi, a duvida tendo surgido da diversidade de notações seguidas.

Quanto á forma $\{17,7, \overline{6}\}$ citada por V ZIMÁNYI encontramos a

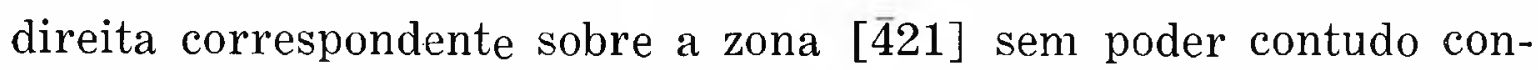
trolal-a; não a cito, por tal motivo, nem mesmo como duvidosa.

Julgamos preferivel seguir criterio diverso do adoptado quer por GOLDSCHMIDT e SCHRÖDER e ZIMÁNYI como por ROSICKÝ, para a orientação dos crystaes. De facto as fórmas negativas $\{12 \overline{1}\}$ e $\{31 \overline{2}\}$, uma direita e outra esquerda (que têm aliás na phenacita do Brasil as suas correspondentes $\{21 \overline{1}\}$ e $\{13 \overline{2}\}$, esquerda e direita respectivamente) são faces de pequena frequencia. Pareceu-nos melhor empregar, para a orientação, entre os rhomboedros de $3 .^{\mathrm{a}}$ ordem, as faces bem desenvolvidas e de grande frequencia, dando optimas imagens, faces essas que orientamos como positivas e assumiram os symbolos $\{20 \overline{1}\}$ e $\{2 \overline{1} 0\}$ Determinadas por esta maneira as zonas caracteristicas, tornava-se facil fixar a posição e calcular os indices de todas as demais.

Si mudarmos a orientação escolhida será de tal ordem a complicação dos indices que só esse facto demonstra as vantagens de criterio adoptado.

Os demais autores tomando como referencias faces differentes chegaram, comtudo, ao mesmo resultado como é facil verificar pela comparação das fórmas predominantes citadas por uns e por outros. Os dois rhomboedros escolhidos $\{20 \overline{1}\}$ e $\{2 \overline{10}\}$, por exemplo, transformar-se-iam, com uma rotação de $60^{\circ}$ em torno do $A_{: 3}$, nos negativos $\{5 \overline{4}\}$ e $\{5 \overline{4} 2\}$, só excepcionalmente determinados e dos quaes apenas encontramos o primeiro, como raro e de difficil verificação. 
$\mathrm{Na}$ phenacita de S. Miguel de Piracicaba a zona [111] é constante e muito desenvolvida dando em alguns casos aos crystaes o habitus prismatico bem pronunciado. $\mathrm{Na}$ maioria das vezes, entretanto, o crescimento em relação ao eixo dos " $z$ " não é predominante e a phenacita se reveste de um habitus achatado com grande multiplicidade de fórmas rhomboedricas.

O prisma mais desenvolvido é o de $2 .^{\mathrm{a}}$ ordem, apresentando-se com todas as suas faces em todos os exemplares observados, sem excepção. O de $1 .^{\text {a }}$ ordem é tambem bastante desenvolvido, mas a sua frequencia inferior á de $\{10 \bar{l}\}$ e o crescimento das faces inferior ao daquelle. Quando se accentúa o habitus prismatico (fig. 1) a differença cresce até attingir o desapparecimento do prisma de $1 .^{\mathrm{a}}$ ordem. Dos prismas de $3 .^{\mathrm{a}}$ ordem pudemos verificar a existencia de $\{3 \overline{1} \overline{2}\}$ (novo para a phenacita desta proce-

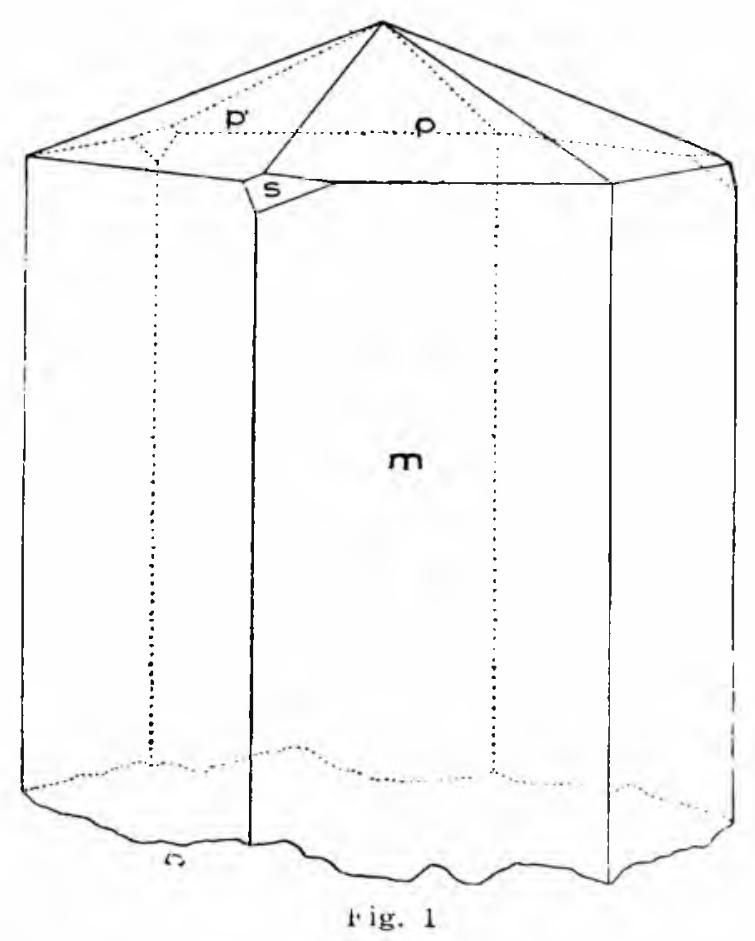

dencia) em pequena faceta de reflexo muito fraco porém nitido. Dois prismas, $\{5 \overline{1} \overline{4}\}$ e $\{5 \overline{4} \overline{1}\}$, novos para a especie, tambem foram por nós assignalados; pudemos controlar a sua determinação por

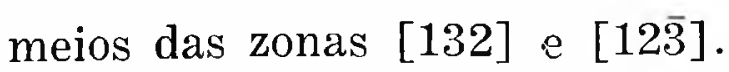

As faces de prisma mostram muitas vezes forte estriatura parallela a [111] dando ao goniometro imagens multiplas que eliminam a precisão das medidas; difficuldade do mesmo genero é provocada pela occurrencia de faces vicinaes. Outras vezes, entretanto, são lisas, brilhantes e bem definidas, com reflexos perfeitos. 
Dos rhomboedros offerecem mais desenvolvimento as faces de $\{210\}$ e $\{201\}$, emprestando aos exemplares o seu habitus caracteristico bi-terminado.

E' commum estarem cobertas por figuras de corrosão bem forte, facilmente visiveis, já descriptas por ZIMÁNYI (11) e assignaladas por RosickÝ (12). Este ultimo autor chama a at'enção para o facto de serem $\{210\}$ e $\{201\}$ desegualmente desenvolvidas; isto acontece, na realidade, em algunș crystaes. Pela observação de maior numero de individuos pudemos, porém, constatar que ora predomina a direita, ora a esquerda e na maior parte dos casos não ha predominancia; podemos, consideral-as esta! isticamente não só de egual persistencia como de egual desenvolvimento.

Dois outros rhomboedro de $2 .^{\mathrm{a}}$ ordem, $\{311\}$ e $\{31 \overline{1}\}$, são facilmente encontrados, mais o direito que o esquerdo: este apresenta, porém, quando medido, faces de desenvolvimento medio e bons reflexos. A fórma $\{31 \overline{1}\}$ em $50 \%$ dos casos nos deu faces do mesmo typo e no restante pequenas facetas de difficil determinação.

De grande importancia, pela frequencia e pelo desenvolvimento, são o rhomboedro fundamental $\{100\}$ e o seu alterno $\{22 \overline{1}\}$, presentes em quasi todos os crystaes e bem desenvolvidos. Entre os rhomboedros de $1 .^{\mathrm{a}}$ ordem figura $\{411\}$ citado apenas por SLAVIK e não citado mas desenhado por GoLDSCHMIDT e SCHRöDER; possue facetas muito finas e está presente na maioria dos exemplares: dá medidas difficeis, mas seu controle é feito em qualquer hypothese por uma das muitas zonas de que faz parte. Já o rhomboedro inverso $\{110\}$ si é, ás vezes, encontrado em facetas muito finas dá-nos na maioria dos casos, faces que, além das dimensões, têm contorno nitido e permittem medidas precisas.

Nas zonas formadas por essas faces, medimos com segurança o anglo formado por (100) e (010) e outros identicos para media $63^{\circ} 24^{\prime}$ Com esse dado calculamos o valor da constante:

$$
\alpha=108^{\circ} 00^{\prime} 53^{1 / 3 \prime}
$$

Partindo do angulo (100) : (2īî), tambem medido com segurança nos crystaes mais perfeitos, estabelecemos directamente a relação parametrica para os eixos da cruz hexagonal

$$
\mathrm{a}: \mathrm{c}=1: 0,6610
$$

proximo ao encontrado por Kokscharow e aos obtidos e confirmados pelos outros autores.

(11) Ob. cit., pg. 102.

(12) Ob. cit., pag. 52. 
Entre os rhomboedros de $3 .^{\mathrm{a}}$ ordem destacam-se as duas fórmas directas $\{201\} \quad 210\}$ muito bem desenvolvidas e, como dissemos atraz, de frequencia muito elevada; faces a contornos nitidos e bem limpidos dão optimas imagens. Tambem os dois rhomboedros $\{2 \hat{1} \overline{1}\}$ e $\{12 \overline{1}\}$, inversos, são dignos de nota e fazem parte das fórmas predominantes no habitus da phenacita dessa jazida. A fórma esquerda não foi encontrada por bôa parte dos mineralogistas que fizeram o estudo morphologico do material da mesma procedencia. Embora não apareça em cerca de metade dos exemplares por nós estudados mostra, todavia, faces de grande desenvolvimento.

Seguem-se, em ordem de importancia, os rhomboedros de $3 .^{\mathrm{a}}$ ordem negativos $\{31 \overline{2}\}$ e $\{13 \overline{2}\}$ assim como $\{43 \overline{1}\}$ e $\{34 \overline{1}\}$

Quanto a $\{312\}$ nada temos a acrescentar, porque foi verificada pelos que nos antecederam a constancia da sua occurrencia. Já $\{13 \overline{2}\}$ é fórma nova para a especie; encontramol-a em facetas nitidas cujos reflexos davam, em certos casos, leituras garantidas.

São noves para a phenacita do Brasil o rhomboedro esquerdo $\{43 \hat{1}\}$ e o correspondente direito $\{34 \overline{1}\}$; ambos aparecem em pequenas facetas de mau reflexo, mas podem ser facilmente controlados pelas zonas dos typos [215], [217] e [111].

Além das fórmas até agora descriptas, as demais occorrem em pequenas facetas, não influindo no habitus do crystal. Apresentamse entre faces bem desenvolvidas, em zonas definidas tambem sem auxilio do goniometro, e é quasi sempre possivel no decorrer das medidas encontrar outras zonas que controlem os indices a ellas attribuidos.

Fazendo rapido resumo de todos os rhomboedros encontrados registramos os elementos que nos asseguraram a determinação dessas fórmas raras ou pela primeira vez constatadas. Os valores angulares são dados na tabella I.

Rhomboedros de $1 .^{\alpha}$ ordem - Entre os directos figuram apenas o fundamental $\{100\}$ e $\{411\}$, já citados.

Entre os inversos temos, além de $\{22 \overline{1}\}\{110\}$, outros dois de muito fraca persistencia, existentes em um só exemplar cada um delles: $\{111\}$ - novo para a phenacita do Brasil e $\{441\}$ - para a especie. 


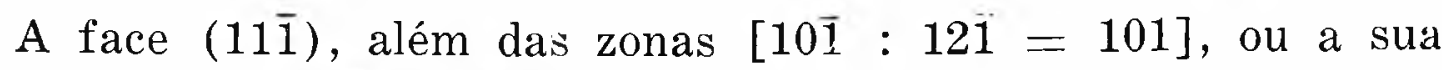
correspondente $[01 \overline{1}: 21 \overline{1}=011]$, encontra-se sobre a zona [110] commum a outras faces de rhomboedros de $1 .^{\mathrm{a}}$ ordem. A face (441). além desta ultima zona é contida na zona [100: $141=01 \overline{4}]$.

Rhomboedros de 2. ${ }^{a}$ ordem - Descriptos, como o foram, os dous pares, $\{210\}$ e $\{201\},\{31 \overline{1}\}$ e $\{3 \overline{1} 1\}$ temos a citar $\{52 \overline{1}\}$ e $\{5 \overline{1} 2\}$, ambos novos para especie.

O primeiro foi encontrado duas vezes, sendo que na segunda em face muito desenvolvida, de contorno rectangular perfeito, for-

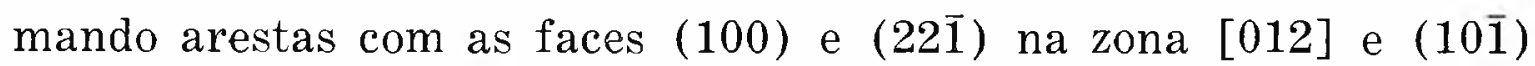

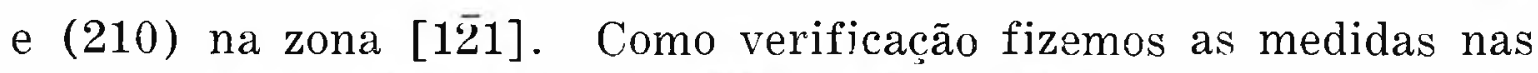
zonas $[11 \overline{2}: 21 \overline{1}=131]$ e $[2 \overline{1} \overline{1}: 41 \overline{1}=1 \overline{1} 3]$.

A fórma inversa foi constatada uma só vez, em faceta pequena. Além da zona [1̄1] pudemos utilizar a zona [221 : $010=102]$.

O rhomboedro directo $\{11,2, \overline{7}\}$, pela primeira vez encontrado na phenacita, é de maior frequencia e menor desenvolvimento. Duas zonas permittiram medidas: [1 $\overline{2} 1]$ comum aos demais de mesmo typo e $[10 \overline{1}: 2 \overline{1} 1=153]$.

O rhomboedro inverso $\{312\}$, tambem novo para o mineral em apreço, foi quatro vezes encontrado e, apezar de fracamente desenvolvido, podemos dal-o com a necessaria certeza visto termos recorrido, além da zona [112] a [210: $102=\overline{2} 41]$.

Ainda ha a accrescentar $\{7 \overline{1}\}\},\{714\}$ e $\{534\}$, que assignalamos sob interrogação, todos sobre a zona [112]. A face (7113) deu-nos uma leitura de $6^{\circ} 23^{\prime}$ para o angulo com (201), havendo uma differença de $16^{\prime}$ para o angulo calculado. (714). com a mesma face, um angulo de $4^{\circ} 59^{\prime}$ para $5^{\circ} 13^{\prime}$ calculado, E a face (534), ainda com (201) o angulo de $17^{\circ} 25^{\prime}$, proximo do valor calculado $17^{\circ}$ $30^{\prime}$ Não nos sendo possivel fazer leitura sobre outras zonas e tratardo-se de facetas de maus reflexos, encontradas uma unica vez, preferimos annotal-as como duvidosas, em particular por não terem sido ainda registradas para a phenacita.

Rhomboedros de $3 .^{a}$ ordem - Já assignalamos anteriormente, $\{20 \overline{1}\},\{2 \overline{1} 0\},\{21 \overline{1}\},\{12 \overline{1}\} \quad\{31 \overline{2}\},\{13 \overline{2}\},\{43 \overline{1}\}$ e $\{34 \overline{1}\}$

Outras duas fórmas $\{41 \overline{1}\}$ e $\{41 \overline{1}\}$ já determinadas por ZIMÁNYI, estavam presentes em mais de um dos crystaes estudados. 


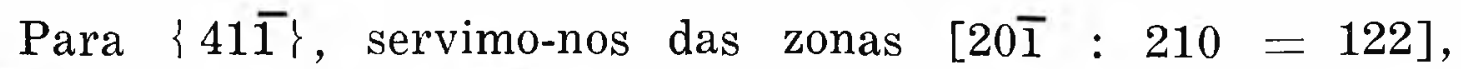
$[01 \overline{1}: 100=011]$ e $[2 \overline{1} \overline{1}: 110=1 \overline{1} 3]$; para $\{4 \overline{1} 1\}$ das correspondentes $[2 \overline{1} 0: 201=12 \overline{2}],[0 \overline{1} 1: 100=011]$ e $[2 \overline{1} \overline{1}: 101=13 \overline{1}]$. Foram as mesmas a que recorreu Zimányi e as leituras feitas ao goniometro foram mais ou menos proximas:

\begin{tabular}{|c|c|c|c|}
\hline & $\mathbf{A}$ & $\mathrm{z} 11$ & 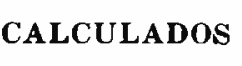 \\
\hline $0 \overline{1} 0):(32 \overline{5} \overline{4})-(\overline{2} 11):(41$ & $53^{\circ} 48^{\prime}$ & - & $\overline{0} \cdot 1^{\circ} \quad 4^{\prime}$ \\
\hline $1 \overline{2} 3):(32 \overline{5} 4)-(210):(41 \overline{1})$ & $16^{\circ} 13^{\prime}$ & $16^{\circ} 17^{\prime}$ & $16^{\circ} 19^{\prime}$ \\
\hline$\overline{1} 1):(32 \overline{5} 4)-(100):(41 \overline{1})$ & $14^{\circ} 56^{\prime}$ & $14^{\circ} 30^{\prime}$ & $14^{\circ} 43^{\prime}$ \\
\hline$(5 \bar{z} \overline{3} 4)-(2 \overline{1} \overline{1}):$ & $53^{\circ} 52^{\prime}$ & $53^{\circ} 44^{\prime}$ & $54^{\circ} \quad 4^{\prime}$ \\
\hline$(201):($ & $16^{\circ} 1^{\prime}$ & ou $16^{\circ} 39^{\prime}$ & $19^{\prime}$ \\
\hline$(5 \overline{2} \overline{3} 4)-(100)$ & $14^{\circ} 45^{\prime}$ & $14^{\circ} 36^{\prime}$ ou $14^{\circ} 48^{\prime}$ & $14^{\circ} 43$ \\
\hline
\end{tabular}

Os dous rhomboedros positivos $\{62 \overline{1}\}$ e $\{6 \overline{1} 2\}$ foram determinados trez vezes cada um. O direito faz parte da relação de ZIMÁNYI, mas o esquerdo é novo para a especie. Fizemos as medidas relativas ao primeiro sobre as zonas $[201: 210=122] \mathrm{e}$ [22̄ $: 100=012]$, as mesmas utilisadas por ZiMÁNYI, obtendo resultados bem proximos; quanto ao segundo utilisamos as zonas $[2 \overline{1} 0: 201=12 \overline{2}]$ e $[100: 2 \overline{1} 2=021]$. Damos abaixo os valores medios:

\begin{tabular}{|c|c|c|c|c|c|c|c|}
\hline \multirow[b]{2}{*}{$(43 \overline{7} 7)$} & \multirow[b]{2}{*}{$:(10 \overline{1} 1)$} & \multirow[b]{2}{*}{$(62 \overline{1})$} & \multirow[b]{2}{*}{$:(100)$} & SALDANHA & \multicolumn{2}{|c|}{ ZIMANYI } & CALCUlado \\
\hline & & & & $15^{\circ} 11^{\prime}$ & $15^{\circ}$ & $3^{\prime}$ & $15^{\circ} \quad 23^{\prime}$ \\
\hline$\overline{1} \overline{-1}$ & : (1123) & $-(62 \overline{1})$ & $(210)$ & $9^{\circ} 56^{\prime}$ & $10^{\circ}$ & $3^{\prime}$ & $10^{\circ} 11 / \underline{2}^{\prime}$ \\
\hline & $(10 \overline{1}$ & $(6 \overline{1} 2)$ & $:(100)$ & $15^{\circ} 13^{\prime}$ & - & - & $15^{\circ}$ \\
\hline & $(2 \overline{1} \overline{1} 3)$ & $-(6 \overline{1} 2)$ & $:(201)$ & $10^{\circ} 15^{\prime}$ & - & - & $10^{\circ} \quad 11 / 2$ \\
\hline
\end{tabular}

A fórma $\{524\}$ é o unico dos rhomboedros inversos (o esquerdo) que correspondem aos direitos $\{20 \overline{1}\}$ e $\{2 \overline{1} 0\}$ Foi assignalado duas vezes e é novo para a phenacita do Brasil (13)

(13) Embora seja discutida a determinação de ( $52 \overrightarrow{4}$ ) feita em crystaes de cutra procedencia preferimos dal-a unicamente como nova para o material de S. Miguel de Piracicaba. 
Apesar de se apresentar em facetas alongadas e finissimas deu valores angulares bem proximos dos calculados.

O rhomboedro positivo direito $\{310\}$ apenas fez parte de um dos crystaes. A sua occurrencia confirma, entretanto, a determinação de Slavik, para esta localidade. Não é commum para os crystaes de outras procedencias e fôra assignalada apenas na phenacita de Framont e dos Montes Ilmen.

Seguem-se tres fórmas de muito fraco desenvolvimento, encontradas duas vezes cada uma e de que pudemos controlar a determinação: $\{85 \overline{1}\},\{831\}$ e $\{14 \overline{3}\}$, ainda não encontradas no mineral em estudo, desta ou de outra localidade. As medidas relativas a $\{85 \bar{\imath}\}$ foram feitas as zonas [221: $210=\overline{1} 22] \mathrm{e}$ $[110: 2 \overline{1} \overline{1}=1 \overline{1} 3]$. Para $\{831\}$ utilizamos $[01 \overline{1}: 210=122]$ e $[2 \overline{1} \overline{1}: \overline{1} 43=1 \overline{5} 7]$. A face $(14 \overline{3})$ encontramol-a sobre as zonas [011 : $110=\overline{1} 11]$ e $[10 \overline{1}: 02 \overline{1}=212]$.

Os demais rhomboedros de $3 .^{\mathrm{a}}$ ordem encontrados não nos é possivel dal-os com segurança. Quanto a $\{651\}$, embora as medidas fossem feitas sobre duas zonas $[110: 101=111] \mathrm{e}$ [210:12 $\overline{1}=124]$, as imagens fracas e diffusas não nos deram a certeza precisa; o mesmo devemos dizer sobre $\{78 \overline{3}\}$, em relação a $[22 \overline{1}: 120=2 \overline{1} 2]$ e $[210: 121=\overline{1} 23]$. Já $\{812\},\{27 \overline{5}\}$ e $\{10,7,1 \overline{1}\}$ não nos permitiram nem mesmo medidas sobre uma segunda zona. Deram-nos, entretanto, aproximações entre os angulos medidos e os angulos calculados, sobre $[100: 2 \overline{1} 2=021]$, $[011: 110=111]$ e $[112: 211=131]$, respectivamente.

\begin{tabular}{|c|c|c|c|c|c|}
\hline & & & & MEDIDO & CALCUlado \\
\hline (1011) & $:(31 \overline{2} 3)$ & $-(100)$ & $:(8 \overline{1} 2)$ & $12^{\circ} \quad 4^{\prime}$ & $11^{\circ} 36^{\prime}$ \\
\hline$(\overline{1} 2 \overline{1} 0)$ & $:(5,12, \overline{7}, 4)$ & $-(011)$ & $:(27 \overline{5})$ & $27^{\circ} \quad 28^{\prime}$ & $27^{\circ} 10^{\prime}$ \\
\hline$(01 \overline{1} 0)$ & $:(17 \overline{6} 2)$ & $-(11 \overline{2})$ & $:(10,7,1 \overline{1})$ & $22^{\circ} 11^{\prime}$ & $22^{\circ} 56^{\prime}$ \\
\hline
\end{tabular}

As innumeras faces vicinaes existentes na phenacita dessas jazidas dão reflexos multiplos, de difficil separação, não só na zona prismatica como tambem proximas aos rhomboedros, em especial nas zonas de menor importancia. 
Dentre as muitas encontradas conseguimos fazer a determinação das pertencentes ás fórmas $\{16,5, \overline{3}\},\{10,3, \overline{2}\},\{10, \overline{2}, \overline{3}\}$ e $\{14, \overline{3}, 4\}$, na vizinhança dos rhomboedros $\{41 \overline{1}\}$ e $\{62 \overline{1}\}$, todos de terceira ordem-positivos.

Sobre a zona $[021: 120=\overline{2} 12]$ medimos as faces $(3,16,5)$ e $(2,10,3)$, controlando-as com as zonas [ $[\overline{1} 43: 134=711]$ e $[221: 12 \overline{1}=4 \overline{1} 6]$, respectivamente. Sobre a zona $[02 \overline{1}: 120$ $=\overline{2} 12]$ fizemos as medidas relativas ás faces $(4,14, \overline{3})$ e $(3,10, \overline{2})$ que tambem puderam dar medidas na zona $[11 \overline{2}: 021=5 \overline{1} 2]-$ a primeira — e na zona $[12 \overline{1}: \overline{1} 22=6 \overline{14}]$ — a segunda.

São os seguintes os valores angulares:

\begin{tabular}{|c|c|c|c|c|c|}
\hline & & & & EDII & ULA \\
\hline & $(\overline{2} 113)$ & $-(\overline{3}, 16,5)$ & $:(021)$ & $11^{\circ} 57^{\prime}$ & $11^{\circ} 30^{\prime}$ \\
\hline & $(\overline{5} 146)$ & $-(\overline{3}, 16,5)$ & $:(\overline{1} 43)$ & $13^{\circ} 57^{\prime}$ & $13^{\circ} 54$ \\
\hline ) & $:(\overline{2} 113)$ & $-(\overline{2}, 10,3)$ & $:(021)$ & $12^{\circ} 25^{\prime}$ & $12^{\circ} 26^{\prime}$ \\
\hline & $(\overline{3} 122)$ & $-(\overline{2}, 10,3)$ & $:(12$ & $14^{\circ} \quad 1^{\prime}$ & $14^{\circ}$ \\
\hline & $(12 \overline{1} 3)$ & $-(3,10,2)$ & : (120) & $12^{\circ} 30^{\prime}$ & $12^{\circ} 26^{\prime}$ \\
\hline 1) & $(1322)$ & $-(3,10,2)$ & $:(121)$ & $14^{\circ} 17^{\prime}$ & $14^{\circ}$ \\
\hline 15) & : $\quad(\overline{2} 113)$ & $-(4,14, \overline{3})$ & $(021)$ & $29^{\circ} \quad 47^{\prime}$ & $29^{\circ} \quad 12^{\prime}$ \\
\hline $7,7,15)$ & $: \quad(121 \overline{3})$ & $-(4,14, \overline{3})$ & $:(120)$ & $13^{\circ} 30^{\prime}$ & $13^{\circ}$ \\
\hline
\end{tabular}

Já vimos anteriormente que pouco varia o habitus da phenacita das jazidas de S. Miguel de Piracicaba, a não ser quando é maior o crescimento na direcção do $A_{3:}$ e, em consequencia, diminue a riqueza de faces rhomboedricas (combinações $n .^{\circ} 1$ e 2 ). Nos crystaes prismaticos achatados que poderiamos dizer de habitus rhomboedricos as combinações variam muito e o numero de faces augmenta nos individuos de pequenas proporções.

$\mathrm{Na}$ tabella II annotamos as diversas combinações e nella podemos ver quaes as fórmas que possuem maior persistencia. Em maior numero as fórmas occorrem num ou noutro crystal e além de raras, são de muito fraco desenvolvimento.

As fórmas que se desenvolvem, sem excepção, em todos os crystaes são: o prisma de $2 .^{a}$ ordem $\{101\}$, os dous rhomboedros 


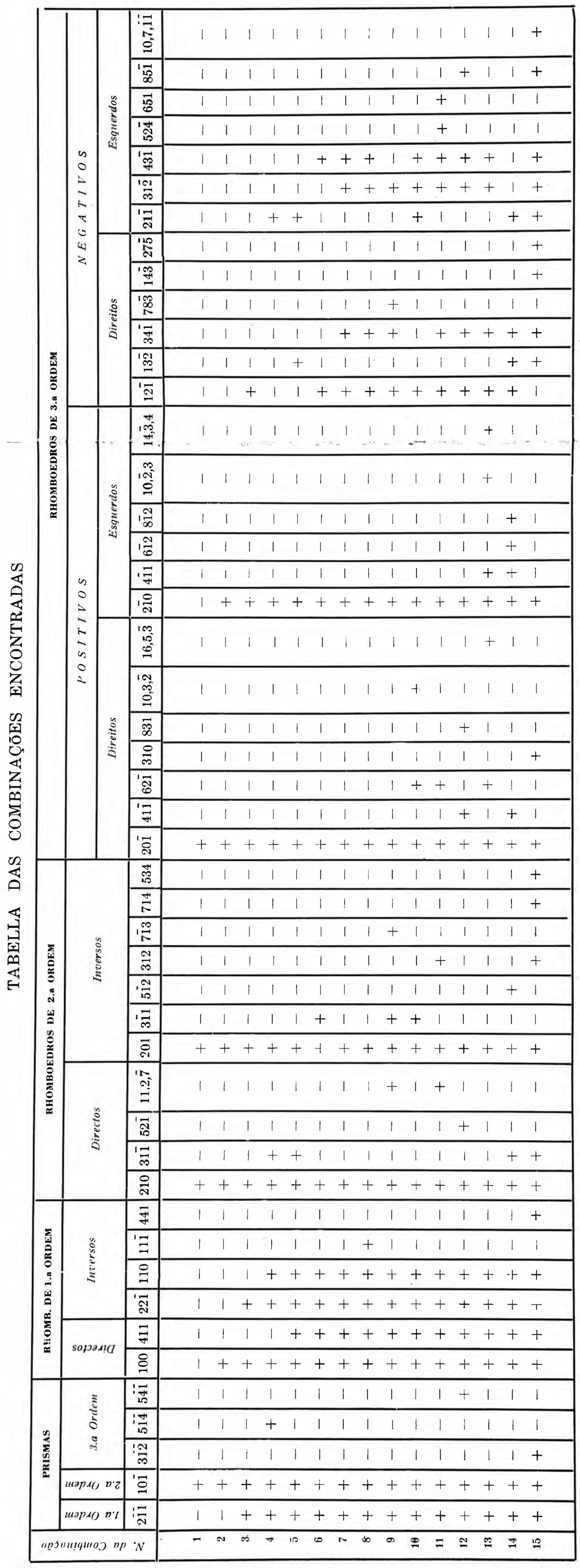


de $2 .^{\mathrm{a}}$ ordem $\{210\}$ e $\{201\}$ e o rhombotdro de $3 .^{\mathrm{a}}$ ordem, positivo, $\{201\}$

O desenvolvimento dessas fórmas é proporcional ás dimensões dos crystaes, com prejuizo muitas vezes da existencia das demais.

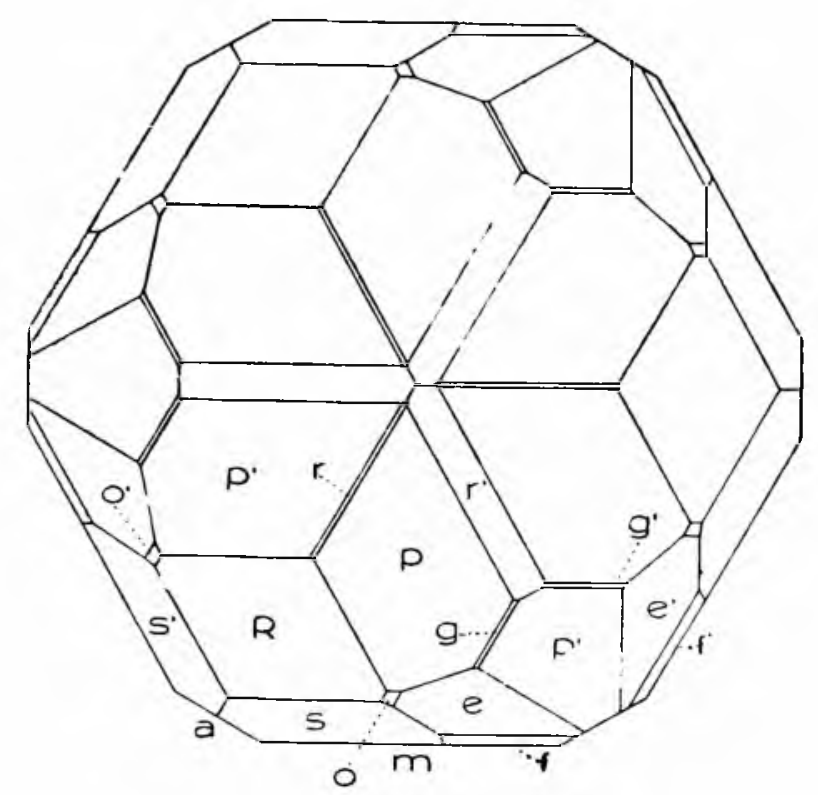

Fig. 2

Na fig. 2 desenhamos, projectadas sobre o plano (111), as fórmas frequentes da phenacita de S. Miguel de Piracicaba, proporcionalmente ao seu desenvolvimento médio, mostrando a distribuição dos rhomboedros mais persistentes e das zonas caracteristicas. 


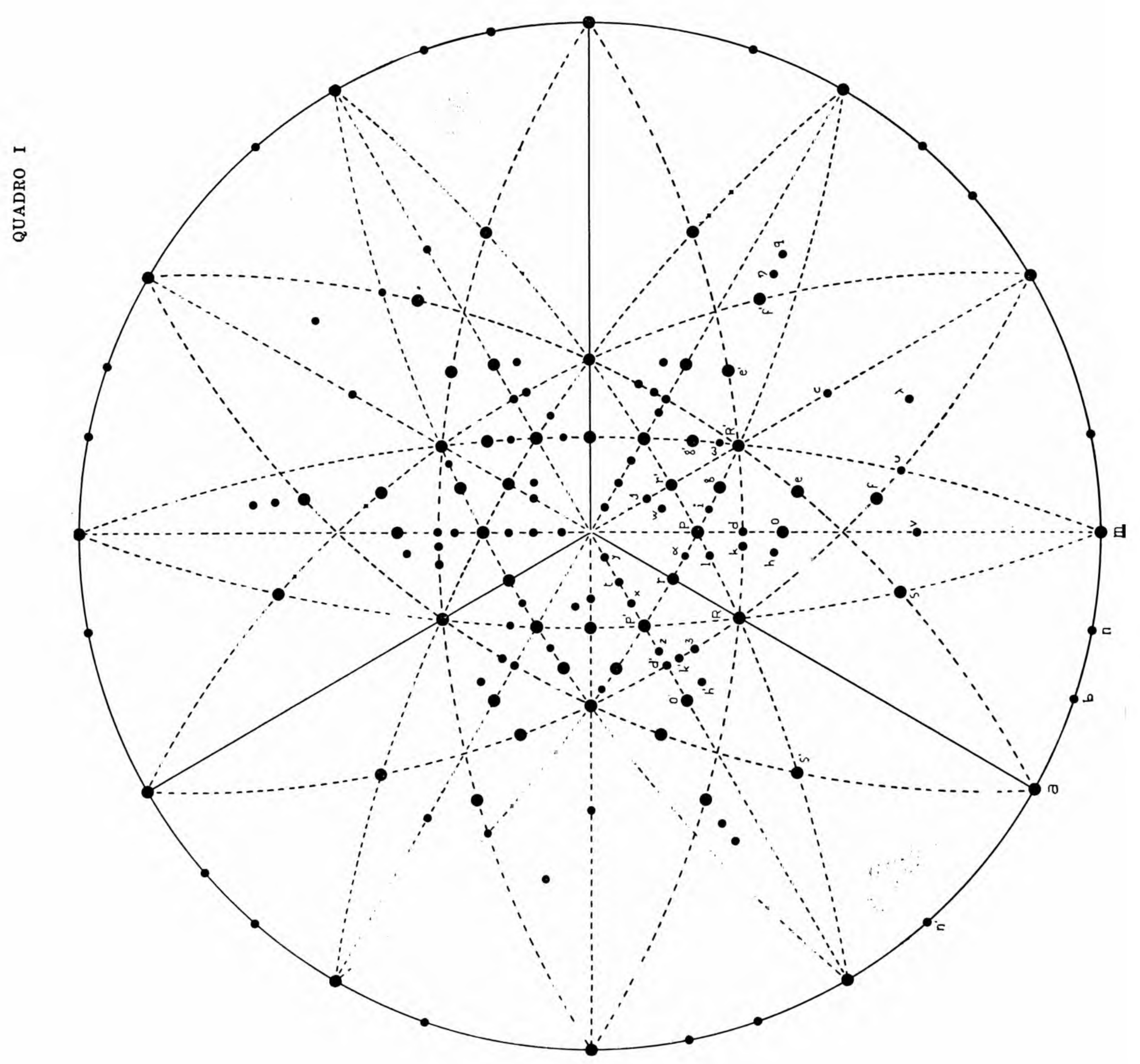


A phenacita é mineral que apresenta na sua crystallisação um grande numero de fórmas, accrescida a relação com as que tivemos a opportunidade de determinar. Foi verificado por PARKER, segundo informa $H$. Brasseur (14), que entre cerca de 700 listas de fórmas citadas por $\mathrm{V}$ GoLDSCHMIDT (15) apenas 28 possuem mais de 100 fórmas. A phenacita não attinge este numero pois sommam 70 todas as determinadas por varios autores (16), em

(14) H. Brasseur - Sur la morphologie de l'azurite - Zeit. f. Krist. 77. 1931, pgs., 177 - 238. Citação na pag. 179.

(16) Além da bibliographia citada nas paginas precedentes (notas $1 \mathrm{e}$ 9), tambem consultamos:

E. S. DANA - A system of Mineralogy - 1837-1868, pgs. 462-463.

C. Hintze - Handbuch der Mineralogie, II Bd., 1897, pgs. 38-43.

BeYrich - Pogg. Ann. 34, 1835, pg. 519; 41, 1837, pg. 323 (GOLDSCHIMIDT, Index Kryst. 2, 1890, pg. 464 .

Rose $u$. Riess -- Pogg. Ann., 69, 1846, pg. 145 (HINTZE, Handbuch, d. Min., 2, pg. 41).

G. Seligmann - Ueber Schweizer Phenakit - Zeit, f. Krist., 13, 188, pg. 65.

Neus Jahrb. f. Min. Geol. u. Pal., 1, 1880, pg. 129.

M. WEBSKY - Ueber das Vorkommen von Phenakit in der Schweiz - Zeit. f. Krist. 7, 1883, pgs. 107-108.

Monatsbeder. d. Berl. Akad., 17. Nov. 1881, S. 1007.

G. Seligmann - Krystallographische Notizen - Zeit. f. Krist., 6, 1882, pg. 102.

W Cross u. W J. Hillebrand - Einige interessante Mineralvorkommen von Pike's Peak, Colorado - Zeit. f. Krist., 7, 1883, pg. 431.

Amer. Journ. Sc. (3) 24, pg. 281. 


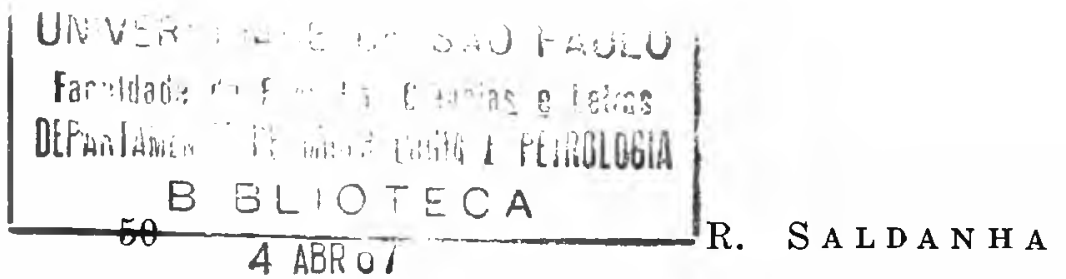

material de diversas procedencias. E' contudo de grande riqueza morphologica, especialmente si levarmos em conta que, dada a sua symetria trigonal e sua classe rhomboedrica, cada fórma possue 6 faces, tanto os prismas como os rhomboedros. Si se tratasse de mineral a symetria mais baixa, como por exemplo a azurita, a um mesmo numero de fórmas não corresponderia uma tal multiplicidade de faces.

G. SeligmanN - Mineralogische Beobachtugen - Zeit. f. Krist., 7, 1883, pgs. 107-108.

W. E. HIDDEN - Mineralogische Notizen - Zeit. f. Krist. 12, 1887, pgs. 506-507.

Amer. Journ. Sc., 32, Sept. 1886, pg. 204.

S. L. Penfield - Phenakit von Colorado - Zeit. f. Krist., 12, 1887, pgs. 635-637.

Amer. Journ. Sc., 33, Feb. 1887, pg. 130.

A. Des Cloizeaux - Note sur la phenacite de Colorado et de Framont - Bul. de la Soc. Fr. de Min., 9, 1886, pgs. 171-175.

S. L. Penfield u. E. S. Sperry - Mineralogische Notizen - Zeit. f. Krist., 17, 1890 , pg. 406 .

Amer. Journ. Sc., 36, Nov. 1888, pgs. 317-331.

P Hautefeille u. A. Perrey - Darstellung von Phenakit und Smaragd Zeit. f. Krist., 18, 1891, pg. 327.

W. S. Yeates - Neue Fundorte von Phenakit - Zeit. f. Krist., 20, 1892, pg. 410 .

Amer. Journ. Sc., 1890, 39, pg. 325-40, pg. 259.

Lackorx - Mineral. de France, 1893-1895, 1, pg. 205.

A. Des Cloizeau et A. Lacroix -- Phenacite de Saint-Christopre-en-Oisans -. Bul. de la Soc. Fr. de Min., 17, 1894, pgs. 33-36.

C. VRBA - Mineralogische Notizcn, VI (Phenakit von Ober Neusattel) Zeit. f. Krist., 24, 1895, pgs. 119-123.

C. Hintze -- Ueber Krystallisirten Phenakit aus Schlesien - Zeit f. Krist., 28. 1897, pgs. 174-176.

H. B̈̈скsтвӧм - Ueber Phenakit von Kragerö - Zeit. f. Krist., 30, 1899, pgs. $352-356$. 
Não dispondo de dados seguros sobre os exemplares de outras jazidas, limitamo-nos a estudar estatisticamente a phenacita de S. Miguel de Piracicaba, com os elementos por nós mesmo colhidos no presente trabalho. São assim unicamente 44 fórmas que intervem na analyse estatistica, despresados tambem os 4 rhomboedros vicinaes que, por se tratar de fórmas que occorrem anormal e esporadicamente, consideramos de persistencia e desenvolvimento nullos

C. H. WARREN - Minerologische Notizen (Pseudomorphosen nach Phenakit von Greenwood, Maine) - Zeit. f. Krist., 30, 1899, pgs. 598-600.

W. C. BRöGger - Hellandit von Lindvikskollen bei Kragerö, Norwegen -Zeit. f. Krist., 42, 1907, 420-421.

L. J. SPENCER - Phenakit und andere Mineralien aus Deustch-Ostafrika Zeit. f. Krist., 45, 1908, pg. 292.

Min. Mag. 14, 1906, pgs. 178-183.

C. PALACHE - Mineralogische Notizen (Phenakit als Unwandlungs product des Danalith von Gloucester, Mass.) Zeit. f. Krist., 44, 1908, pgs. 17-18.

F. Kolbeck u. M. Henglein - Zuei neue Phenakitvorkormmen in Schlesien — Zeit. f. Krist., 49, 1911, pg. 491.

O. C. Farrington u. E. W. Tillotson - Uber verschiedene Mineralien aus der Sammlung in Chicago - Zeit. f. Krist., 48, 1911, pg. 117.

Field Columbian Museum, Geol. Serv. 3, 1908, pgs. 131-163.

W. T. Schaller - Krystallographische Notizen ïber Albit, Phenakit und Neptunit - Zeit. f. Krist., 48, 1911, pgs. 554-556.

F. Kolbeck u. M. Henglein - Ein neues Vorkommen von Phenakit von den Zimmerzgängen von Ehrenfriedersdorf in Erzgebirge - Zeit. f. Krist., 49, 1911, pg. 484.

A. Russel - Uber das vorkommen des Phenakits in Cornwall -- Zeit. f. Krist., 53, 1913, pg. 583.

H. MicheL - Kïnstliche Phenakithrystalle - Zeit. f. Krist., 53, 1913 pg. 538-569.

K. MIELEITNER - Über einige Mineralien von der gregnitz in Fichtelgebirge — Zeit. f. Krist., 56, 1921, pgs. 94-96.

Mineralogische Sammlung des Staates, München.

L. Weber - Neues Vorkommen von Scheweizer Phenakit - Zeit f. Krist., 59, 1924, pgs. 493-503. 
Nos estudos estatisticos de diversos mineraes realisados por P. Niggli (18), R. L. PARker (19), H. BiÄsch (20), H. BrasSEUR (21) e F. RoDolico (22) a persistencia geral de uma fórma foi calculada pelo numero de vezes que figurava nas combinações encontradas, desde que não possuiam dados mais completos sobre as determinações feitas por outros autores em relação aos mesmos mineraes.

Dispondo nós de todos os elementos sobre a phenacita de S. Miguel de Piracicaba, não nos pareceu justo tal criterio na apreciação da importancia relativa de cada fórma no desenvolvimento do mineral.

De facto, duas fórmas que persistem em todas as combinações podem ter valores differentes na morphologia do crystal porque emquanto uma desenvolve quasi todas as suas faces a outra pode apresentar apenas um pequeno numero destas em cada exemplar.

Achamos assim, assim, preferivel calcular a persistencia geral dividindo o numero de faces que uma fórma apresentava pelo total $6 \mathrm{n} \quad(\mathrm{n}=$ numero de crystaes examinados $)$ de vezes em que essa fórma deveria apparecer si possuisse todas as suas faces desenvolvidas.

E' facil a exemplificação na phenacita do Brasil. Tres rhomboedros, $\{210\},\{201\}$ e $\{201\}$, persistem em todas as combinacooes encortradas. No entretanto, si os de $1 .^{\text {a }}$ ordem mostram o crescimento de quasi todas as faces, o de $3 .^{a}$ raramente pode mostrar mais de 3 ou 4 das 6 faces que theoricamente deveriam

(17) Encontramos uma noticia geral sobre os estudos estatisticos e bom resumo bibliog'raphico no trabalho de R. L. FARKER - Die Kristallmorphologie im Lichte neuerer analytische Unter'suchungen - Fortschritte d. Min., Krist. u. Pet., 1930, pags. $75-139$.

(18) .P NigGi - Kristalisation and Morphologie des rhombischen Schivefels - Zeit. f. Krist., (Festband) 58, 1923, pgs. 490521.

(19) R. L. PARKer - Zw Kristallographie von Anatas und Rutil Zeit. f. Krist., (Festband) 58, 1923, pgs. 522582.

(20) H. BÏ̈SCH - Morphologische Untersuchung am Hämatit, unter besonderer Beriicksichtigung des Vorkommens vom Piz Cavradi (Tavetsch) Zeit. f. Krist., 70, 1929, pgs. 1159.

(21) H. BRA:SEUR - Ob. cit.

(22) F. Rodolico - Studi sulla datolite della formazione ofiolitica Per. di Min., Rcma, 1933 (N. 1), pgs. 114. 
apparecer. Emquanto a persistencia dos primeiros, segundo o nosso criterio, é egual a 85 e 88,3 , respectivamente, a do ultimo não excede 55.

Claro que nem sempre será possivel seguir uma tal orientação. A não ser para o material de uma só jazida em que o estudioso disponha dos exemplares examinados, não será exequivel obter os dados necessarios sobre crystaes de differentes origens e, em épocas diversas, estudados por diversos autores. Não ha duvida, porém, de que este systema de observação dá estatisticamente resultados muito mais completos e precisos.

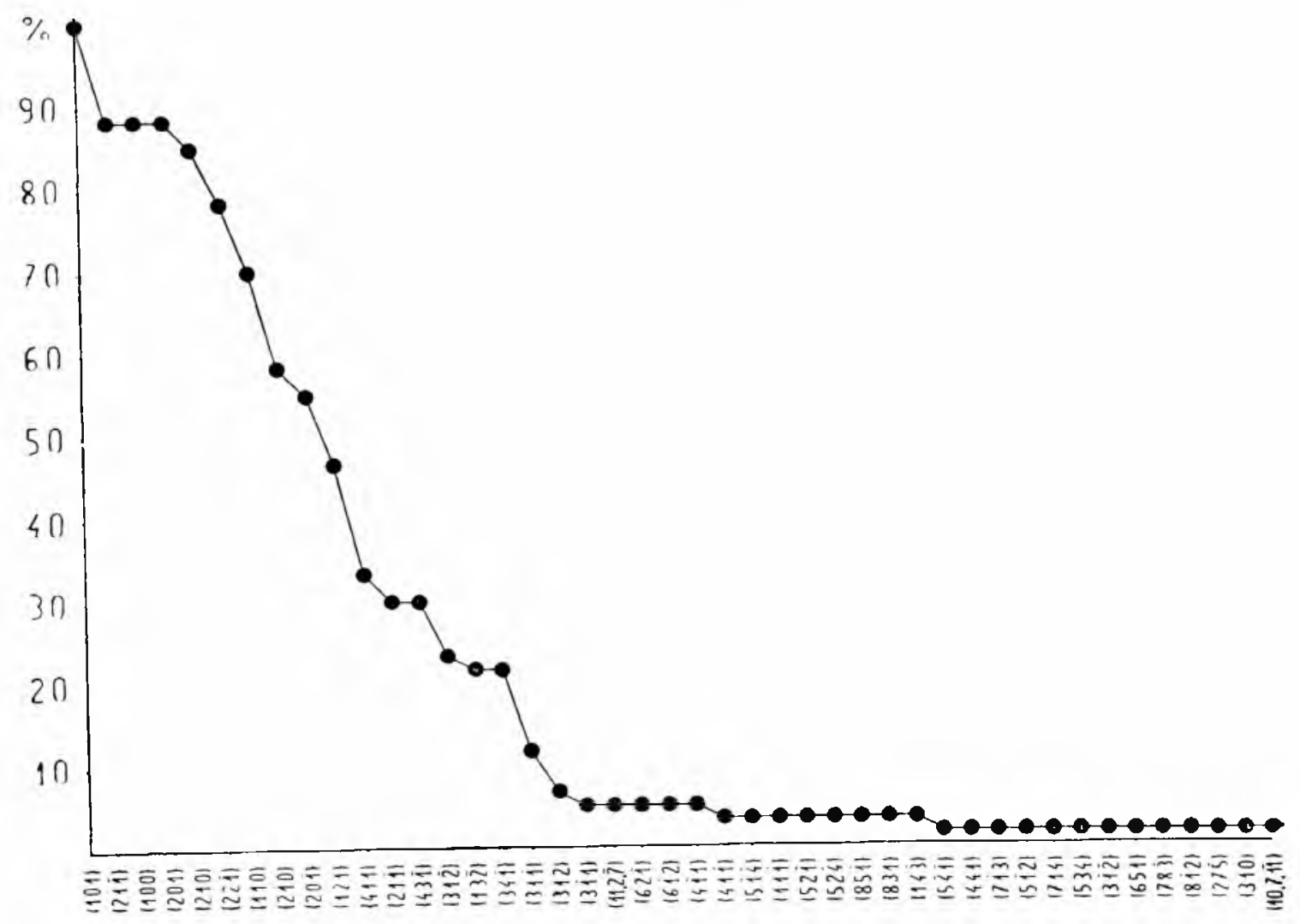

Fig. 3

$\mathrm{Na}$ figura 3 temos a curva das persistencias das 44 fórmas.

Ao primeiro exame verificamos que, feita a comparação com as de outros mineraes estudados (23), a natureza da curva se aproxima bastante daquella da azurita, pela distribuição mais ou menos regular ao longo da linha, com uma differença maxima, entre dous pontos consecutivos, de 13,3 unidades. A segunda metade da curva é, porém, situada nas proximidades do eixo das abcissas, e quasi a elle parallelo, em tres degráos mais ou menos longos. E' a porção da curva que corresponde ás fórmas raras ou de baixa persistencia, fórmas que não influem no habitus do mi-

(23) Obs. cits. de P. Niggli, R. L. Parker, H. Biäsch, H. Brasseur e F. RODOLICO. 
neral nem se revestem de maior importancia morphologica. Ao contrario, aquellas que estão situadas na primeira metade da curva mereceram um cuidado especial na analyse dos valores estatisticos.

Além do prisma de $2 .^{a}$ ordem $\{101\}$, a persistencia maxima $(100 \%)$, havia um grupo de fórmas a alta persistencia (entre 80 e $90 \%$ ) e mais um grupo a persistencia media (entre 40 a $80 \%$ ). Restava ainda o grupo a baixa persistencia (de 10 a $40^{\circ}$ ) e o grupo numeroso de fórmas a persistencia minima $\left(<10^{\circ}\right)$ que já vimos acima.

Da observação do graphico assim construido, não tiramos conclusões que nos satisfizessem. Realmente, fórmas como $\{411\}$ e $\{43 \overline{1}\}$ que possuiam faces sem maior importancia no habitus dos crystaes, figuravam na curva ao lado de $\{211\}$ que se destacara pelas dimensões e perfeição nos crystaes em que occorrera. Por outro lado, o rhomboedro fundamental collocava-se na mais alta posição após $\{10 \overline{1}\}$, quando de facto, em todos os exemplares, as

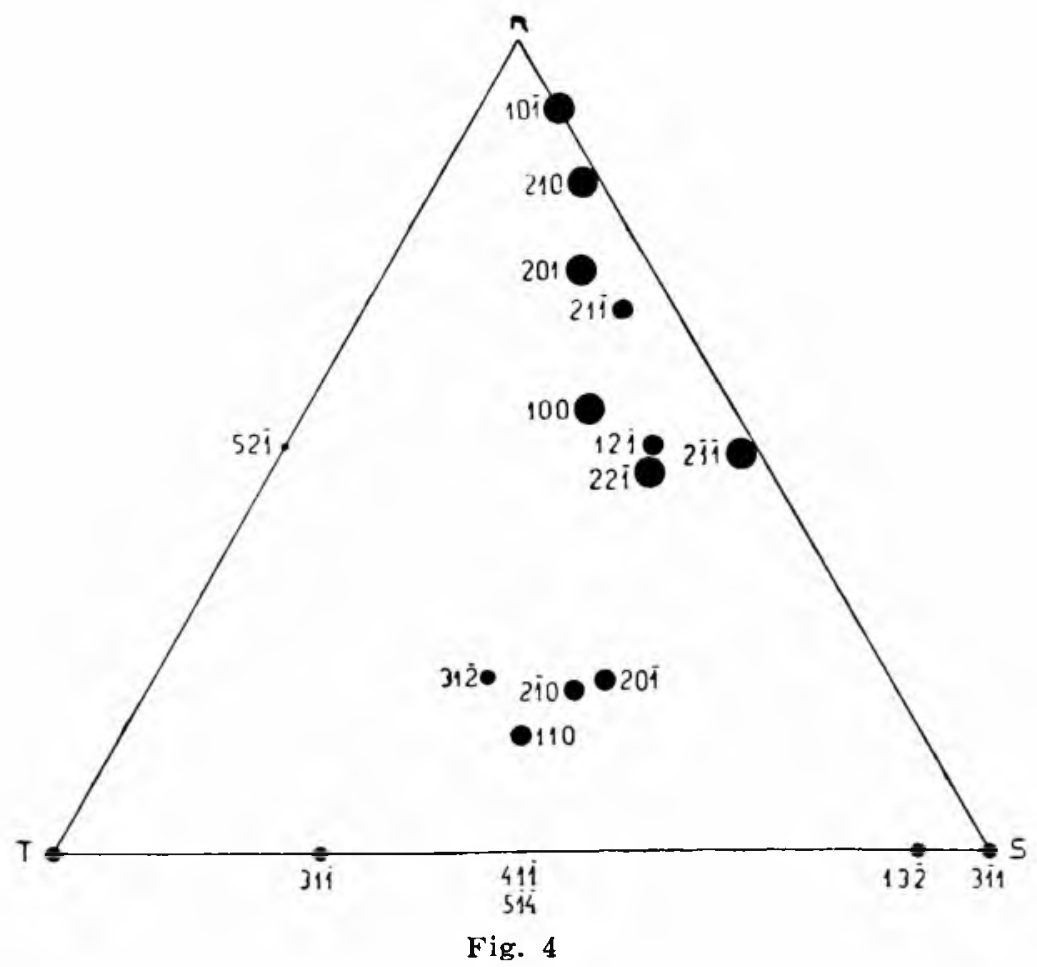

fórmas predominantes eram $\{210\}$ e $\{201\}$, como já haviamos constatado.

Resolvemos, então recorrer ao estudo do desenvolvimento das fórmas, utilizando as expressões de PARKER (24).

(24) R. L. PARKer - Ob. cit. (na Forts. d. Min. Kr. u. Pet., 1930, pg. 87). 


$$
R=\frac{100 . r}{r+s+t} \quad S=\frac{100 . s}{r+s+t} \quad T=\frac{100 . t}{r+s+t}
$$

funcções que independem da frequencia (25) e dão, por comparação, uma ideia do tamanho medio de uma fórma.

O diagrama triangular (fig. 4), em que os vertices $R, S$ e $T$ correspondiam a $100 \%$ de desenvolvimento grande, médio ou pequeno, registrou graphicamente os resultados obtidos.

Proximas ao vertice " $R$ " estavam situadas as fórmas $\{101\}$, $\{210\}$ e $\{201\}$, realmente de grande importancia no habitus de phenacita do Brasil. A seguir entretanto, já se davam inversões na collocação de outras, si comparavamos esse graphico com o das persistencias. O rhomboedro $\{21 \overline{1}\}$, por exemplo, tinha uma posição acima de $\{100\}$ e o seu correspondente $\{121\}$ acima de $\{22 \overline{1}\}$ e $\{2 \overline{1} \overline{1}\}$, fórmas de maior influencia no mineral em apreço. Por outro lado $\{411\}$ estava collocada exactamente no vertice $\mathrm{T}$ emquanto a sua frequencia, como já tivemos occasião de mostrar, era bem elevada.

Encontravamos as mesmas contradicções em um graphico (fig. 5) que registrava os valores resultantes do calculo com a fórmula indicada por BRAsseur (26) :

$$
\mathrm{M}=\frac{(3 \mathrm{r}+2 \mathrm{~s}+\mathrm{t}) 100}{3(\mathrm{r}+\mathrm{s}+\mathrm{t})}
$$

que tambem independe da frequencia.

As fórmas $\{3 \overline{1} \overline{2}\},\{52 \overline{1}\}$ e outras mais, revestiam-se de uma importancia que na realidade não possuiam emquanto $\{110\}$, $\{20 \overline{1}\}$ e $\{210\}$, para citar algumas, passavam a posições secundarias.

Qualquer methodo que desprezasse a noção de persistencia, deixava de traduzir o papel que uma fórma exerce no habitus do mineral pois uma face póde excepcionalmente apresentar um desenvolvimento grande ,mesmo se tratando de fórma rara, passando a figurar nos graphicos resultantes em situação de relevo. O desen-

(25) ${ }^{\prime} r$ " $s$ e "t são, respectivamente, o numero de vezes que a fórma se apresenta em faces de desenvolvimento grande, medio ou pequeno.

(26) H. Brasseur - Ob. cit., pg. 230. 
vclvimenio pequeno das faces que pertençam a uma fórma frequente não significa, por sua vez, que a sua influencia no habitus seja despresivel; entretanto, quer no de PARKer quer no de Brasseur, avizinhavam-se ou coincidiam com as fórmas de quasi nenhuma importancia.

Havia, portanto, forte discordancia entre os resultados obtidos em funcção unicamente da persistencia ou unicamente do desenvolvimento.

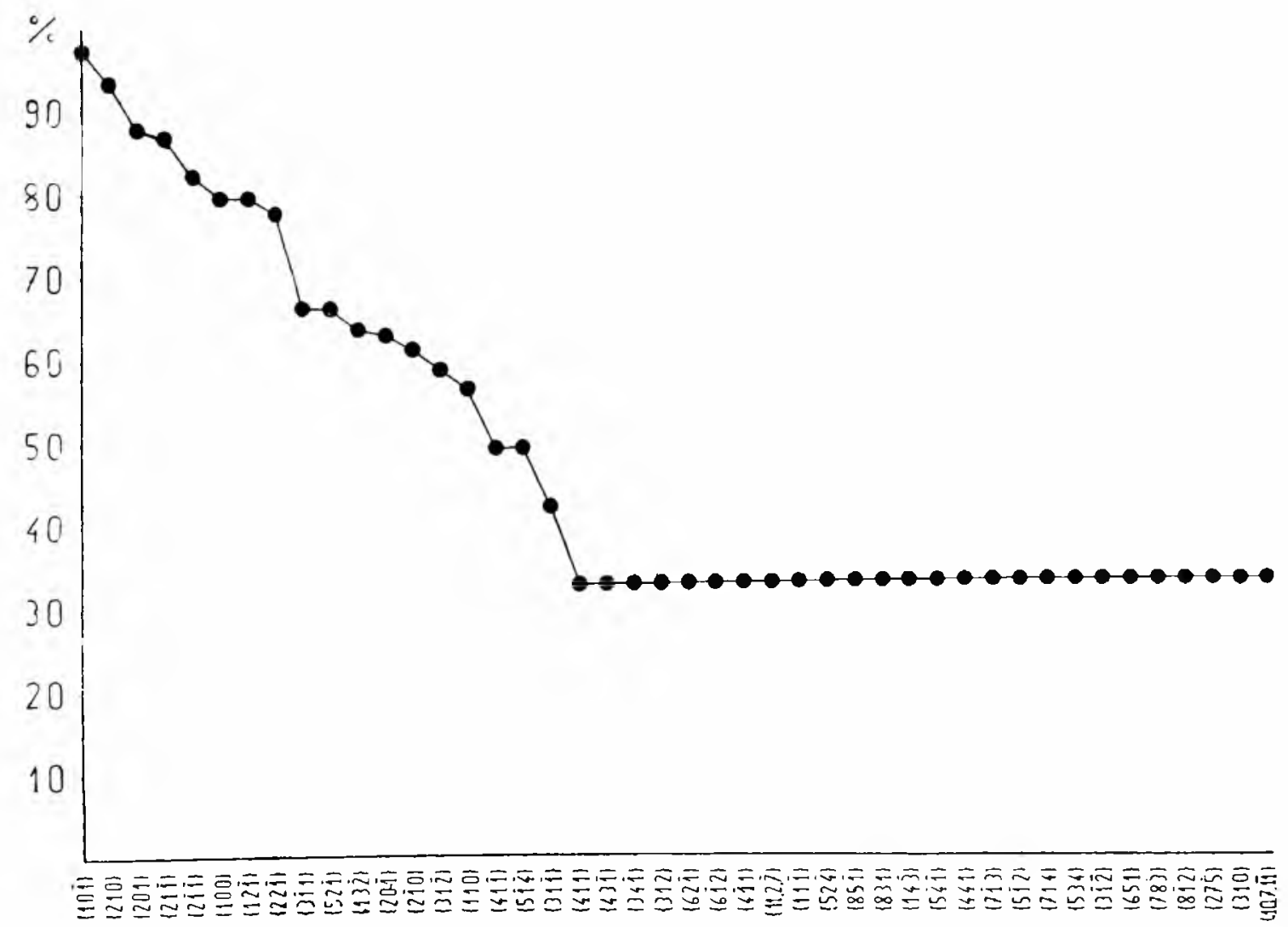

Fig. 5

Era preciso procurar a expressão que dependesse ao mesmo tempo dos dous factores para chegar a uma conclusão decisiva. O methodo de GoLDSCHMIDT (27) resolvia o problema, pois reduz em dados numericos a noção conjunta da dimensão e da frequencia, sendo ao mesmo tempo qualitativo e quantitativo, por meio da fórmula proposta:

(27) Em R. L. PARker, ob. cit. (na Forts. d. Min. Kr. u. Pet., 1930), pgs. 86 e 87.

Em H. Brasseur, ob. cit., pgs. 229 e 230. 


$$
\mathrm{G}=\frac{3 \mathrm{r}+2 \mathrm{~s}+\mathrm{t}}{3 \mathrm{n}}
$$

em que apenas multiplicamos, de accordo com o estabelecido desde $o$ inicio, o numero " $n$ " de crystaes observados pelo numero 6 de faces que uma fórma deve theoricamente apresentar em cada crystal de phenacita calculando os valores em percentagens:

$$
G=\frac{(3 r+2 s+t) 100}{3 \times 6 n}
$$

Para a construcção do graphico correspondente os valores, relativos ás diversas fórmas, foram obtidos pela multiplicação dos já

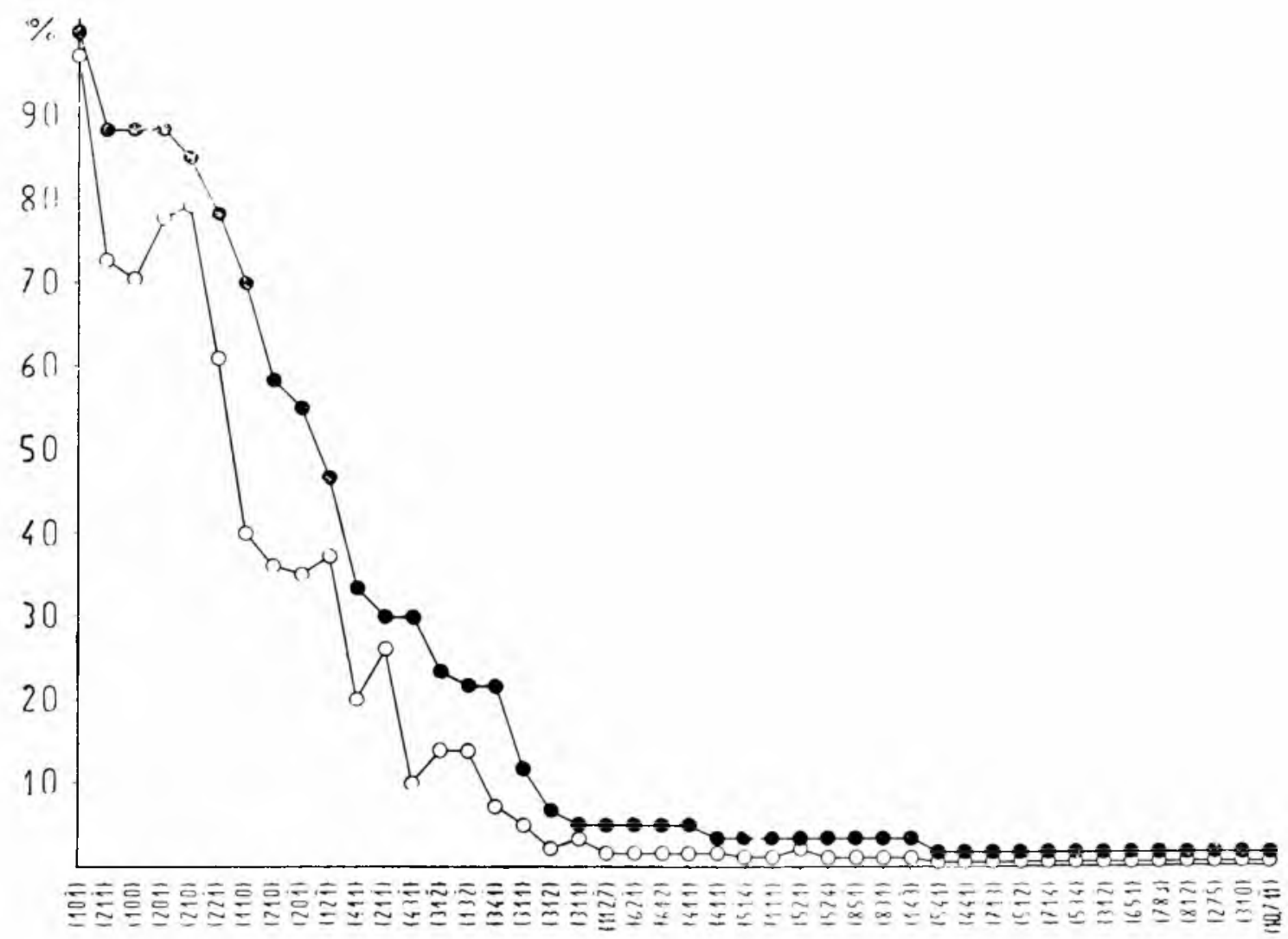

l'ig. (;

determinados valores " $P$ " da persistencia e "M" da grandeza média segundo BRASSEUR; é facil verificar que

$$
\mathrm{G}=\mathrm{M} \times \mathrm{P}
$$

si dermos a " $\mathrm{P}$ " a expressão 


$$
\mathrm{P}=\frac{\mathrm{r}+\mathrm{s}+\mathrm{t}}{6 \mathrm{n}}
$$

Construimos, em primeiro logar, o graphico sobre um outro identico ao da fig. 3. No eixo das ordenadas o valor 100 corresponderia ao de uma forma que se apresentasse com as 6 faces, todas de desenvolvimento grande, em todos os " $n$ " crystaes observados.

A' primeira vista verificava-se (fig. 6) que ás fórmas $\{2 \overline{1} \overline{1}\}$, $\{100\},\{221\}$ e $\{110\}$ correspondia uma fórte queda, accentuada em $\{411\},\{43 \overline{1}\}$ e $\{34 \overline{1}\}$; por outro lado $\{201\}$ e $\{210\}$ ascendiam na curva e diminuia a differença entre os dous rhomboedros de $3 .^{\mathrm{a}}$ ordem, negativos, $\{21 \overline{1}\}$ e $\{12 \overline{1}\}$ Todas as transformações traduziam a verdade constatada na observação feita durante o demorado estudo dos crystaes, confirmando que o criterio dessa vez seguido era o mais aconselhavel.

Construimos finalmente o graphico dos valores obtidos com a fórmula de Goldschmidt (fig. 7).

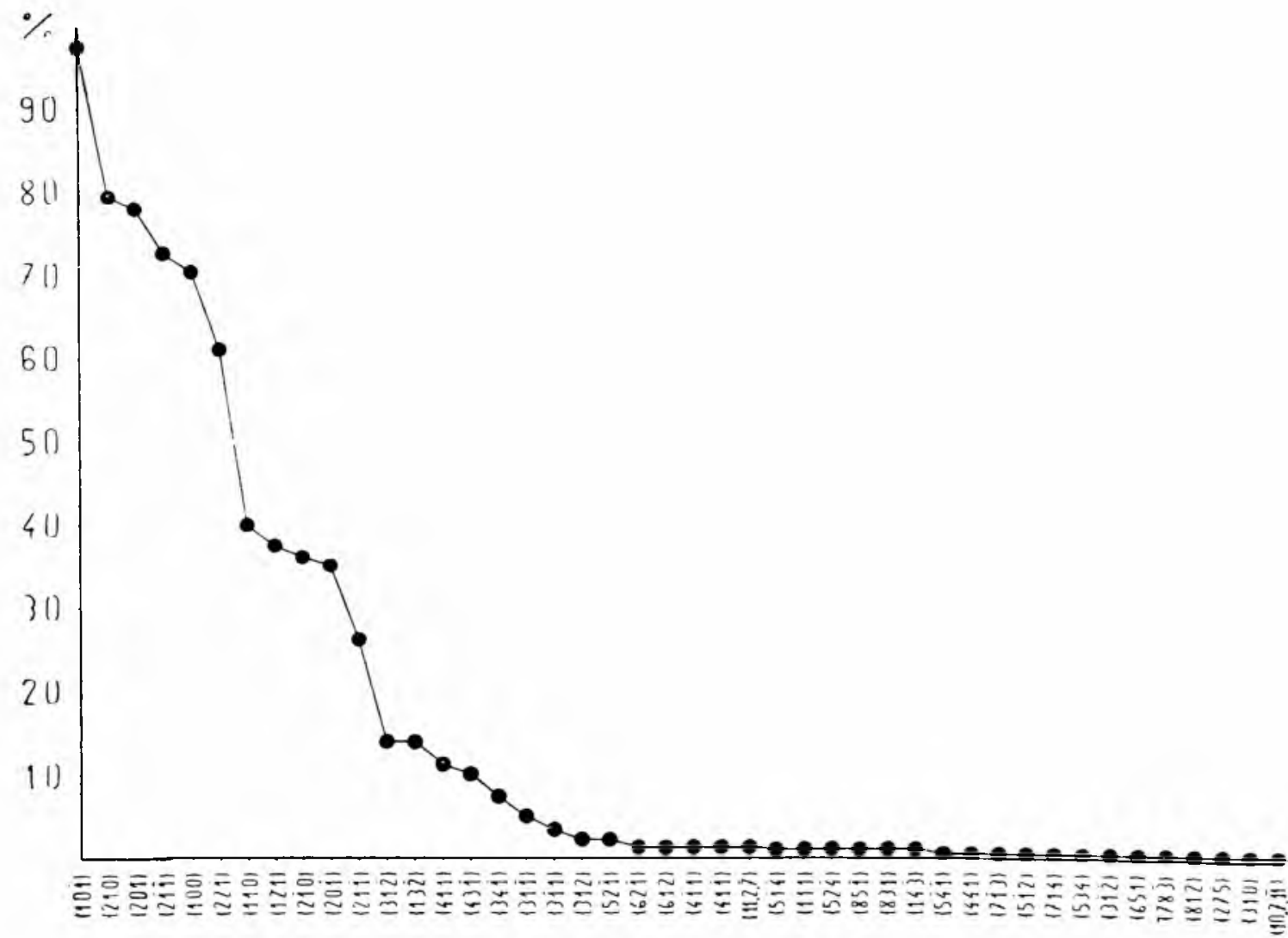

Fig. 7

(28) Lembramos mais uma vez que, em nosso trabalho, substituimos sempre o valor de "n" pelo de " $6 \times n$ " 
Separado em quatro zonas de 100 a 90,90 a 40,40 a 10 e 10 a 0 , ellas conservavam uma divisão semelhante á adoptada na curva das persistencias, comprehendendo a segunda os grupos de fórmas a alta e media persistencia. Corrigia-se, porém, na nova curva a posição das fórmas que nos difficultavam as conclusões, podendo-se então acceitar o graphico como representação de que fôra realmente observado.

$\mathrm{Na}$ primeira zona figurava sósinho o prisma de $2 .^{\mathrm{a}}$ ordem $\{10 \overline{1}\}$ com 97,2 de persistencia-desenvolvimento, destacando-se como de facto já o observaramos, pela frequencia e dimensões das suas faces.

$\mathrm{Na}$ segunda zona, em ordem decrescente, vinham os dois rhomboedros de $2 .{ }^{\mathrm{a}}$ ordem, $\{210\}$ e $\{201\}$, o prisma de $1 .^{\mathrm{a}}$ ordem $\{2 \overline{1} \overline{1}\}$ e os dois rhomboedros de $1 .^{a}$ ordem $\{100\}$ - fundamental e $\{22 \overline{1}\}$ - inverso correspondente. Na phenacita de São Miguel de Piracicaba verificamos, não somente nós como os demais autores que a estudaram, são principalmente os dous prismas e os quatro rhomboedros já citados que predominam, destacando-se entre estes ultimos os dous de $2 .^{\mathrm{a}}$ ordem.

$\mathrm{Na}$ terceira zona apareciam na parte superior o rhomboedro de $1 .^{\mathrm{a}}$ ordem, inverso, $\{110\}$ e os dous pares de rhomboedros de $3 .^{\mathrm{a}}\{2 \overline{1} 0\}$ e $\{20 \overline{1}\}-$ positivos - e $\{211\}$ e $\{12 \overline{1}\}$ - negativos. $\mathrm{Na}$ parte inferior o rhomboedro de $1 .^{\mathrm{a}}$ ordem, directo $\{411\}$ e os dois de $3 .^{\mathrm{a}}$ negativos, $\{31 \overline{2}\}$ e $\{13 \overline{2}\}$

$\mathrm{Na}$ quarta zona figuravam, com certo destaque, os pares $\{43 \overline{1}\},\{34 \overline{1}\}-$ de $3 .^{\mathrm{a}}$ ordem, negativos $-\{31 \overline{1}\},\{311\}-$ de $2 .^{a}$ ordem; dahi por diante a curva se aproximava exageradamente do eixo das abcissas.

Podemos resumir a licção do graphico, de accordo com as observações feitas em todos e em cada um dos exemplares. As fórmas de maxima e grande persistencia-desenvolvimento $(>40)$ são as fórmas predominantes no habitus da phenacita do Brasil. As de persistencia-desenvolvimento médio $(<40>10)$ são fórmas que influem no habitus sem nelle predominar; a influencia é tanto maior quanta mais proximas ficam do limite superior. As fórmas a persistencia-desenvolvimento pequeno nada modificam no habitus do crystal a não ser, em certos crystaes, as 4 por nós destacadas.

Vejamos como se distribuem as 48 fórmas (inclusive as consideradas convencionalmente a persistencia e desenvolvimento nullos) pelos quatro typos de fórmas de classe rhomboedrica. 
A tabella III mostra-nos a distribuição para o total das fórmas, para as fórmas em que o valor de "G" é maior que 10 e para as fórmas em que o valor de "G" é maior que 40 .

Os rhomboedros de $3 .^{a}$ ordem predominam entre as 48 fórmas encontradas e ainda entre as 14 de persistencia-desenvolvimento entre 10 e 40, para desaparecer totalmente entre as predominantes. Os rhomboedros de $2 .^{\mathrm{a}}$ ordem sāo mais numerosos que os de $1 .^{\mathrm{a}}$ no conjunto, mas inferiores entre as fórmas influentes no habitus. Finalmente os primas estão sempre em inferioridade na primeira e na segunda divisão.

TABELLA I I I

\begin{tabular}{|c|c|c|c|c|c|c|}
\hline & \multicolumn{2}{|l|}{$\leq 10$} & \multicolumn{2}{|l|}{$>10$} & \multicolumn{2}{|c|}{$>40$} \\
\hline & $\begin{array}{c}\text { Numero } \\
\text { de } \\
\text { Fórmas }\end{array}$ & \% & $\begin{array}{c}\text { Numero } \\
\text { de } \\
\text { Fórmas }\end{array}$ & $\because$ & $\begin{array}{l}\text { Fórmas } \\
\text { de } \\
\text { Numero }\end{array}$ & $\because r$ \\
\hline PRISMAS & 5 & 10.4 & 2 & 14.3 & 2 & 33.3 \\
\hline $\begin{array}{c}h+k+l=o \\
\text { RHOMB. DE } 10^{\mathrm{a}} \text { ORDEM } \\
\{\text { hll }\} \text { ou }\{\text { hhl }\}\end{array}$ & 6 & 12.5 & 4 & 28.6 & 2 & 33.3 \\
\hline $\begin{array}{c}\text { RHOMB. DE } 3 .^{\mathrm{a}} \text { ORDEM } \\
\mathrm{h}+\mathrm{k}+1=3 \mathrm{r}\end{array}$ & 11 & 22.9 & 2 & 14.3 & 2 & 33.3 \\
\hline $\begin{array}{c}\text { RHOMB. DE } 2 .^{\mathrm{a}} \text { ORDEM } \\
\mathrm{h}+\mathrm{k}+\mathrm{l} \gtrless 3 \mathrm{~K}\end{array}$ & 25 & 54.2 & 6 & 42.8 & - & - \\
\hline
\end{tabular}

Entre as fórmas predominantes a distribuição entre prismas e rhomboedros de $1 .^{\mathrm{a}}$ e $2 .^{\mathrm{a}}$ ordem é absolutamente egual e o habitus da phenacita do Brasil depende da distribuição e desenvolvimento das suas faces.

No conjuncto das fórmas, entretanto, apezar da superioridade numerica dos rhomboedros de $3 .^{\mathrm{a}}$ ordem, ha um certo equilibrio que podemos verificar si fizermos as sommas dos valores de $\mathrm{G}$.

Prismas

Rhomboedros de $1 .^{\text {a }}$ ordem

Rhomboedros de $2 .^{\mathrm{a}}$ ordem

Rhomboedros de $3 .^{\mathrm{a}}$ ordem
172,0

153,8

171,7

189,0

Como ultima observação podemos assignalar a não correspondencia entre as fórmas directas e inversas, na sua totalidade. o que destaca a symetria da phenacita, pertencente á classe rhomboedrica. 


\section{DISCUSSÃO DAS ZONAS}

Para a discussão da importancia das zonas dous criterios vêm sendo seguidos, sob o ponto de vista estatistico.

O primeiro consiste em calcular a distribuição das fórmas nas diversas zonas, contando o numero de fórmas presentes em cada uma dellas, para recalculal-o em percentagens referidas ao numero total de fórmas que o mineral apresenta. Deixamos de utilizal-o porque, como já foi verificado por outros autores, não dá uma bôa traducção da importancia da zona.

Parte o segundo da determinação da importancia da zona em base da expressão

$$
\mathrm{I}=\Sigma \mathrm{n} \mathrm{P}
$$

em que "P" é a persistencia de uma fórma e " $n$ " o numero de suas faces que se encontram na zona (30)

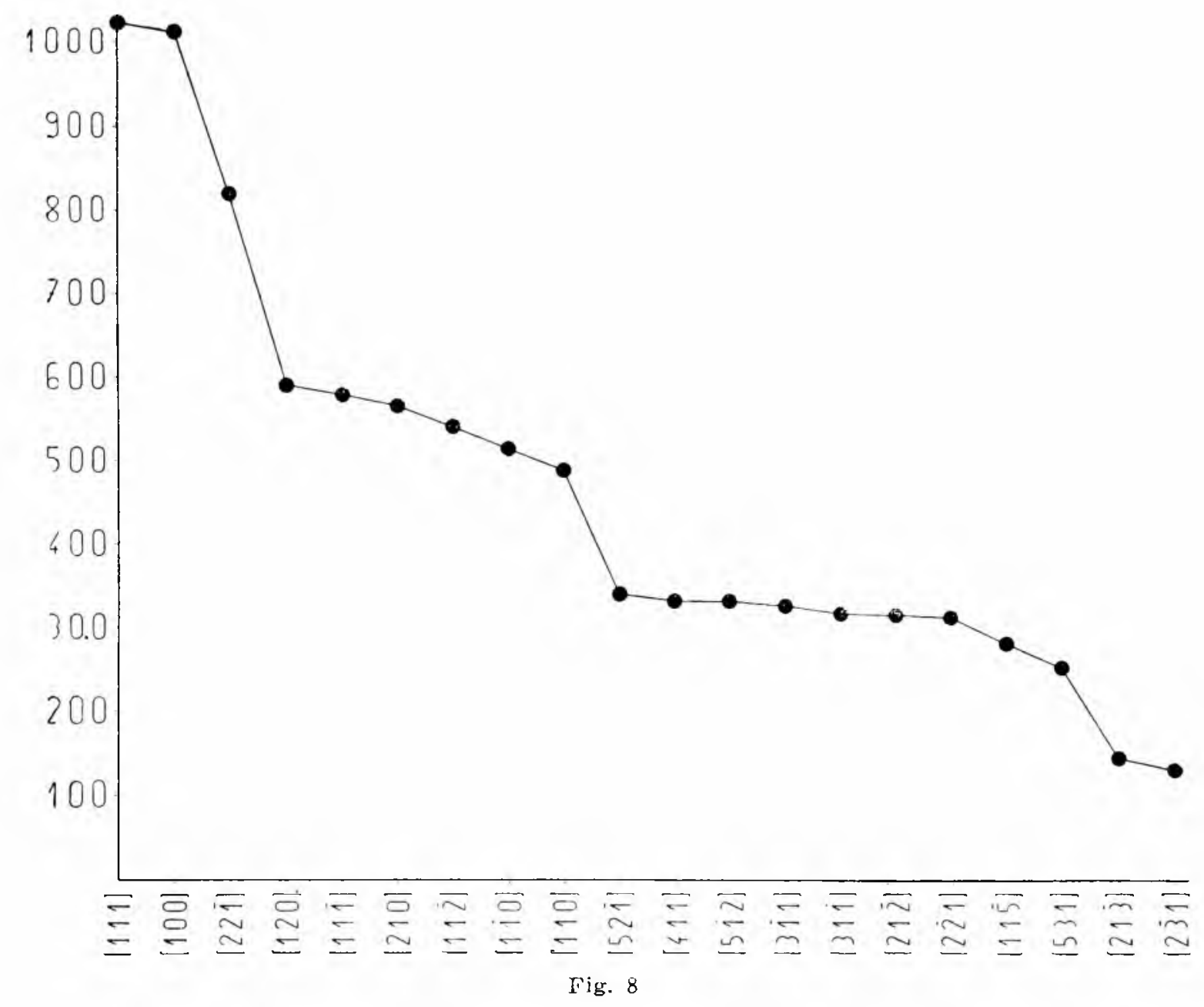

(29) Indicamos com $\Sigma$ que deve ser effectuada a somma dos productos " $\mathrm{nP}$ " das fórmas contidas na zona.

(30) Concordamos com F. Rodolico (ob. cit. pg. 11) na preferencia dada ao methodo de Brasseur, em substituição ao de Bï̈sch. 
De accordo com as conclusões tiradas do estudo das persistencias e desenvolvimentos substituimos o valor de " $\mathrm{P}$ ", na expressão, pelo valor de $\mathrm{G}$ :

$$
\mathrm{I}=\Sigma \mathrm{nG}
$$

Calculado "I" para as diversas zonas e transportados os mesmos resultados nas ordenadas do graphico (fig. 8) correspondente, observamos a sua distribuição.

A zona prismatica [111] e o grupo de zonas rhomboedricas [100] sobrelevavam-se em posição isolada. São as zonas predominantes e caracteristicas dos crystaes de S. Miguel de Piracicaba.

Mais abaixo, com uma forte differença em relação a [100], destacava-se o grupo [221] de zonas symetricas áquellas, ambos os grupos caracterisando o habitus do mineral na sua bi-terminação rhomboedrica.

A [221] ] que um pouco se aproxima das demais podemos denominar grupos de zonas caracteristicas secundarias, para dizer

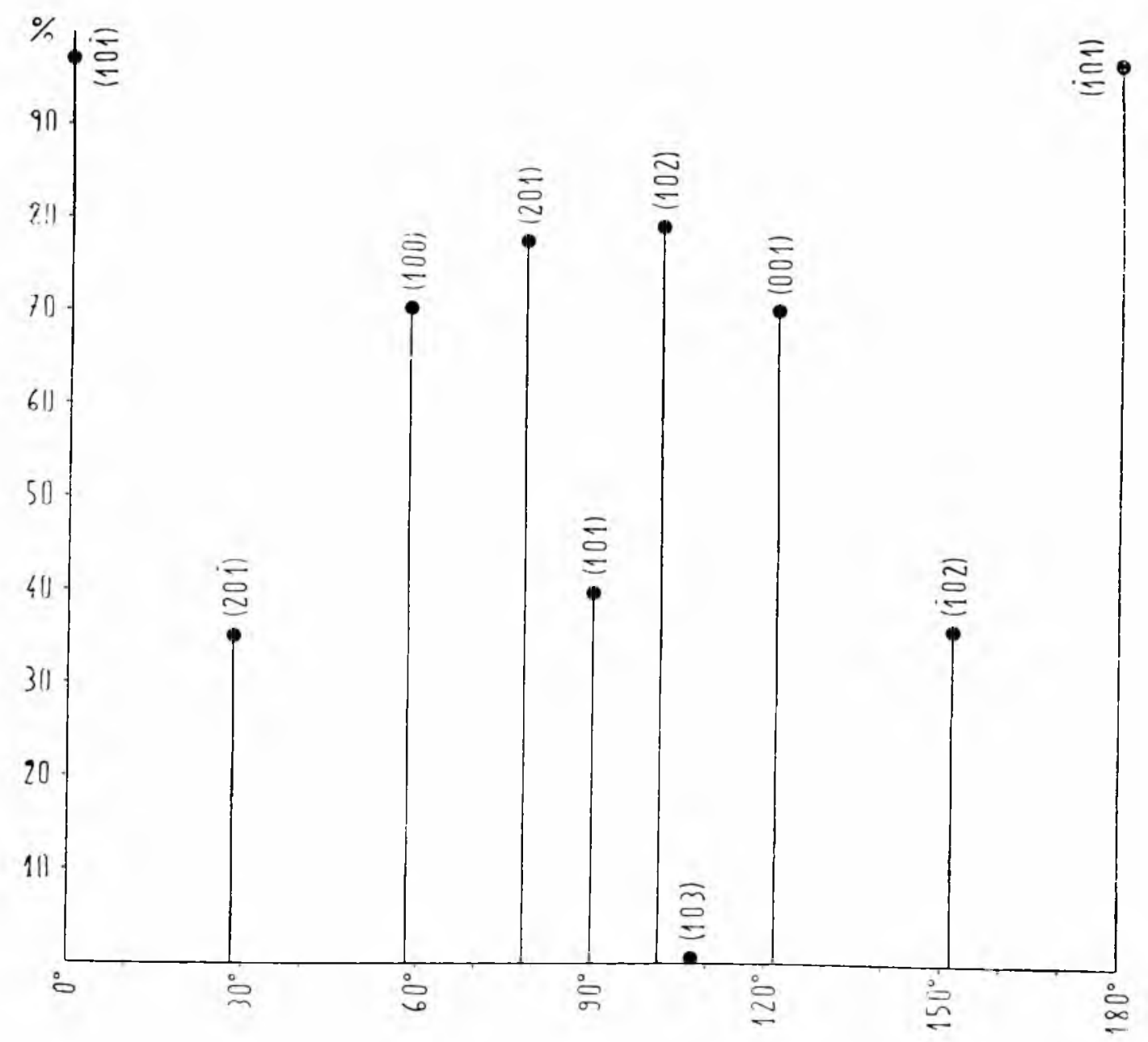

Fig. 9 
TABELLA IV

\begin{tabular}{|c|c|c|c|c|}
\hline \multirow{2}{*}{$\begin{array}{l}\text { GRUPOS DE } \\
\text { ZONAS }\end{array}$} & \multirow{2}{*}{$\begin{array}{l}\text { N." de faces } \\
\text { contidas } \\
\text { na zona }\end{array}$} & \multirow[b]{2}{*}{ FORMAS A QUE PERTENCEM AS FACES } & \multicolumn{2}{|c|}{ VALOR DE } \\
\hline & & & $\begin{array}{l}\text { Sem as } \\
\text { faces de } \\
\text { prisma }\end{array}$ & $\begin{array}{l}\text { Com as } \\
\text { faces de } \\
\text { prisma }\end{array}$ \\
\hline [111] & 30 & $\{\overline{2} \overline{1}\}^{6},\{1 \overline{01}\}^{6},\{3 \overline{1} \overline{2}\}^{6},\{5 \overline{1} \overline{4}\}^{6},\{5 \overline{4} \overline{1}\}^{6}$ & $\ldots$ & 1023,26 \\
\hline$[100],[010]$ e $[001]$ & 18 & $\{20 \overline{1}\}^{2},\{100\}^{4},\{201\}^{2},\{110\}^{2},\{210\}^{2},\{310\}^{2},\{2 \overline{1} 0\}^{2},\{101\}^{2}$, & 819,94 & 1014,38 \\
\hline$[22 \overline{1}],[2 \overline{1} 2]$ e $[\overline{1} 22]$ & 24 & $\begin{array}{l}\{52 \overline{4}\}^{2},\{22 \overline{1}\}^{4},\{4 \overline{1} 3\}^{2},\{8 \overline{7}\}^{2},\{201\}^{2},\{411\}^{2},\{831\}^{2},\{210\}^{2}, \\
\{85 \overline{1}\}^{2},\{43 \overline{1}\}^{2},\{10 \overline{1}\}^{2},\end{array}$ & 623,28 & 817,72 \\
\hline [120], [201] e [012] & 16 & $\{2 \overline{1} 0\}^{2},\{2 \overline{1} 1\}^{2},\{2 \overline{2} 1\}^{2},\{52 \overline{1}\}^{2},\{62 \overline{1}\}^{2},\{100\}^{2},\{3 \overline{2} 1\}^{2},\{2 \overline{11}\}^{2}$ & 445,50 & 591,04 \\
\hline$[1 \overline{1} \overline{1}],[\overline{1} 11]$ e $[\overline{1} 11]$ & 26 & $\begin{array}{l}\{31 \overline{2}\}^{2}=\{21 \overline{1}\}^{2},\{43 \overline{1}\}^{2},\{110\}^{4},\{312\}^{2},\{651\}^{2},\{4 \overline{1} 3\}^{2},\{211\}^{2}, \\
\{3 \overline{2} 1\}^{2},\{7 \overline{5} 2\}^{2},\{4 \overline{3} 1\}^{2},\{101\}^{2},\end{array}$ & 385,48 & 579,92 \\
\hline$[210],[102]$ e $[021]$ & 20 & $\begin{array}{l}\{20 \overline{1}\}^{2},\{2 \overline{1}\}^{2},\{22 \overline{1}\}^{2},\{5 \overline{1} 2\}^{2},\{6 \overline{1} 2\}^{2},\{8 \overline{1} 2\}^{2},\{100\}^{2},\{312\}^{2}, \\
\{52 \overline{4}\}^{2},\{2 \overline{1} \overline{1}\}^{2},\end{array}$ & 421,04 & 566,58 \\
\hline$[1 \overline{12}],[\overline{12} 1]$ e $[\overline{2} 11]$ & 24 & $\begin{array}{l}\{11,2, \overline{7}\}^{2},\{3 \overline{1}\}^{2},\{52 \overline{1}\}^{2},\{210\}^{2},\{534\}^{2},\{312\}^{2},\{714\}^{2},\{201\}^{2}, \\
\quad\{7 \overline{1} 3\}^{2},\{5 \overline{1} 2\}^{2},\{\overline{31} 1\}^{2},\{10 \overline{1}\}^{2},\end{array}$ & 347,68 & 542,12 \\
\hline$[1 \overline{1} 0],[01 \overline{1}]$ e $[\overline{1} 01]$ & 16 & $\{100\}^{2},\{411\}^{2},\{211\}^{2},\{441\}^{2},\{110\}^{2},\{22 \overline{1}\}^{2},\{111 \overline{1}\}^{2},\{2 \overline{1} \overline{1}\}^{2}$, & 369,96 & 515,50 \\
\hline$[110],[101]$ e $[011]$ & 20 & $\begin{array}{l}\{11 \overline{1}\}^{+},\{2 \overline{1} 1\}^{2},\{3 \overline{1} 1\}^{2},\{4 \overline{1} 1\}^{2},\{100\}^{2},\{41 \overline{1}\}^{2},\{31 \overline{1}\}^{2},\{21 \overline{1}\}^{2}, \\
\{10 \overline{1}\}^{2}\end{array}$ & 295,50 & 489,94 \\
\hline$[5 \overline{2} \overline{1}],[\overline{21} 5]$ e $[\overline{1} 51]$ & 10 & $\{31 \overline{1}\}^{4},\{201\}^{2},\{43 \overline{1}\}^{2},\{2 \overline{1} \overline{1}\}^{2}$, & 145,54 & 341,08 \\
\hline [411], [141] e [114] & 8 & $\{3 \overline{1} 1\}^{2},\{22 \overline{1}\}^{2},\{31 \overline{1}\}^{2},\{10 \overline{1}\}^{2}$, & 138,88 & 333,32 \\
\hline$[\overline{5} \overline{2}],[25 \overline{1}]$ e $[\overline{1} 25]$ & 12 & $\{3 \overline{1} 1\}^{4},\{4 \overline{1} 3\}^{2},\{210\}^{2},\{8 \overline{1} 2\}^{2},\{2 \overline{1} \overline{1}\}^{2}$ & 187,74 & 333,28 \\
\hline$[3 \overline{1} 1],[13 \overline{1}]$ e $[\overline{1} 13]$ & 14 & $\{4 \overline{1} 1\}^{2},\{5 \overline{1} 2\}^{4},\{110\}^{2},\{411\}^{2},\{2 \overline{1} 1\}^{2},\{2 \overline{1} \overline{1}\}^{2}$ & 182,20 & 327,74 \\
\hline$[3 \overline{1}],[\overline{1} 13]$ e $[\overline{1} 31]$ & 22 & $\begin{array}{l}\{41 \overline{1}\}^{2},\{5 \overline{2}\}^{4},\{85 \overline{1}\}^{2},\{110\}^{2},\{714\}^{2},\{411\}^{2},\{310\}^{2},\{21 \overline{1}\}^{2}, \\
\{10,7, \overline{1} \overline{1}\}^{2},\{2 \overline{1} \overline{1}\}^{2},\end{array}$ & 172,16 & 317,70 \\
\hline$[21 \overline{2}],[\overline{1} \overline{2} 2]$ e $[\overline{2} 21]$ & 12 & $\{20 \overline{1}\}^{2},\{41 \overline{1}\}^{2},\{62 \overline{1}\}^{2},\{210\}^{2},\{534\}^{2},\{110\}^{2}$, & 816,62 & 316,62 \\
\hline$[2 \overline{2} 1],[1 \overline{2} \overline{2}]$ e $[\overline{2} 12]$ & 10 & $\{2 \overline{10}\}^{2},\{4 \overline{1} 1\}^{2},\{6 \overline{12}\}^{2},\{201\}^{2},\{110\}^{2}$ & 314,40 & 314,40 \\
\hline$[1 \overrightarrow{15}],[\overline{15} 1]$ e $[\overline{5} 11]$ & 8 & $\{411\}^{4},\{312\}^{2},\left\{10 \overline{1}^{-}\right\}^{2}$ & 88,88 & 283,32 \\
\hline$[531],[315]$ e $[153]$ & 10 & $\{11,2, \overline{7}\}^{4},\{2 \overline{1} 1\}^{2},\{31 \overline{2}\}^{2},\{2 \overline{1} \overline{1}\}^{2}$, & 108,86 & 254,40 \\
\hline$[21 \overline{3}],[\overline{13} 2]$ e $[\overline{3} 21]$ & 8 & $\{5 \overline{1} \overline{4}\}^{2},\{20 \overline{1}\}^{2},\{310\}^{2},\{2 \overline{1} 1\}^{2}$ & 145,54 & 146,64 \\
\hline$[2 \overline{3} 1],[1 \overline{2} \overline{3}]$ e $[\overline{3} 12]$ & 12 & $\{5 \overline{4} \overline{1}\}^{2},\{2 \overline{1} 0\}^{2},\{8 \overline{1} \overline{2}\}^{2},\{85 \overline{1}\}^{2},\{21 \overline{1}\}^{2},\{52 \overline{4}\}^{2}$ & 129,98 & 131,08 \\
\hline
\end{tabular}


T A B E L L A V

ZONA $[010]^{*}$

\begin{tabular}{|c|c|c|c|}
\hline Faces & Formas & Valor de G & Observações \\
\hline$(10 \overline{1})$ & $\{101\}$ & 97.22 & \\
\hline$(201)$ & $\{201\}$ & 35 . & 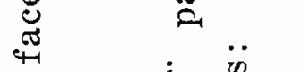 \\
\hline$(100)$ & $\{100\}$ & 70.55 & $\overbrace{0}^{2} \overbrace{0}^{n}$ \\
\hline$(201)$ & $\{201\}$ & $77 \quad 77$ & 哥 $\ddot{0}$ \\
\hline$(101)$ & $\{110\}$ & 40 . & ¿ \\
\hline$(102)$ & $\{210\}$ & 79.44 & $\therefore \&$ \\
\hline$(103)$ & $\{310\}$ & 0.55 & 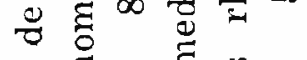 \\
\hline$(001)$ & $\{100\}$ & 70.55 & $\underset{\mathbb{E}}{\mathbb{E}}$ \\
\hline$(\overline{102})$ & $\{2 \widehat{10}\}$ & 36.11 & छี 용 $\frac{0}{\pi}$ \\
\hline$(\overline{101})$ & $\{100\}$ & 97.22 & \\
\hline
\end{tabular}

* Da tabella e do graphico apenas constam as faces da metade superior.

melhor do relevo de [100], observavel nos proprios desenhos (Figs. 1 e 2 ).

As zonas que se seguem, especiaes pela posição que occupam e pela funcção que exercem no desenvolvimento dos crystaes, são a cada momento necessarias durante o trabalho ao goniometro. $\mathrm{Na}$ realidade o seu papel não é tão importante quanto o daquellas que denominamos caracteristicas, si desprezarmos no computo do "I" a ellas relativo a persistencia-desenvolvimento do prisma de $1 .^{\mathrm{a}}$ ou do prisma de $2 .^{\mathrm{a}}$ ordem cujas faces lhes pertencem. Na tabela IV a columna 3 assim calculada apresenta differenças mais fortes que a columna 5 que dá os valores levados ao graphico.

Isso resalta mais visivel estudando em particular zonas de uma e de outra especie.'

Vejamos a zona caracteristica [010]. Das faces de rhomboedros que sobre ella se dispoem, em numero de dezeseis, dez pertencem a fórmas predominantes $(\mathrm{G}>40)$ e quatro a fórmas influentes $(G>10)$ muito proximas ao limite superior; apenas duas são de persistencia-desenvolvimento minima. O graphico (fig. 9) e a tabella respectiva dão uma ideia egual e mais clara do que se passa.

Si determinarmos a persistencia-desenvolvimento media dos rhomboedros que nella figuram encontramos o valor 51,24. Nenhuma outra zona poderá dar média que se aproxime dessa, prova indiscutivel da predominancia da primeira. 
Já para [1]1] , uma dentre as varias zonas especiaes, a apreciação do cáso é inteiramente diversa (fig. 10). Possue maior riqueza de faces, mas das vinte e quatro fases a ella pertencentes apenas duas, do rhomboedro $\{110\}$, fazem parte daquellas que influem no habitus. Outras seis são fórmas a pequena persistenciadesenvolvimento e as restantes fórmas a persistencia-desenvolvimento minima. O valor médio de " $G$ " não ultrapassa 16,06 .

Qualquer zona da mesma especie apresentará aspecto identico, com predominancia de rhomboedros a baixa ou minima persistencia-desenvolvimento e valor médio proximo a 20 .

Assim é possivel constatar, que, apezar de não existir um grande intervallo entre as zonas especiaes e as caracteristicas cabe a estas, muito em particular ás do grupo [100], figurar com re'evo em todos os crystaes da phenacita do Brasil. E' interessante notar que a forte predomnancia de [100] não dá logar á occurrencia de fórmas raras ou menos desenvolvidas a ella pertencentes.

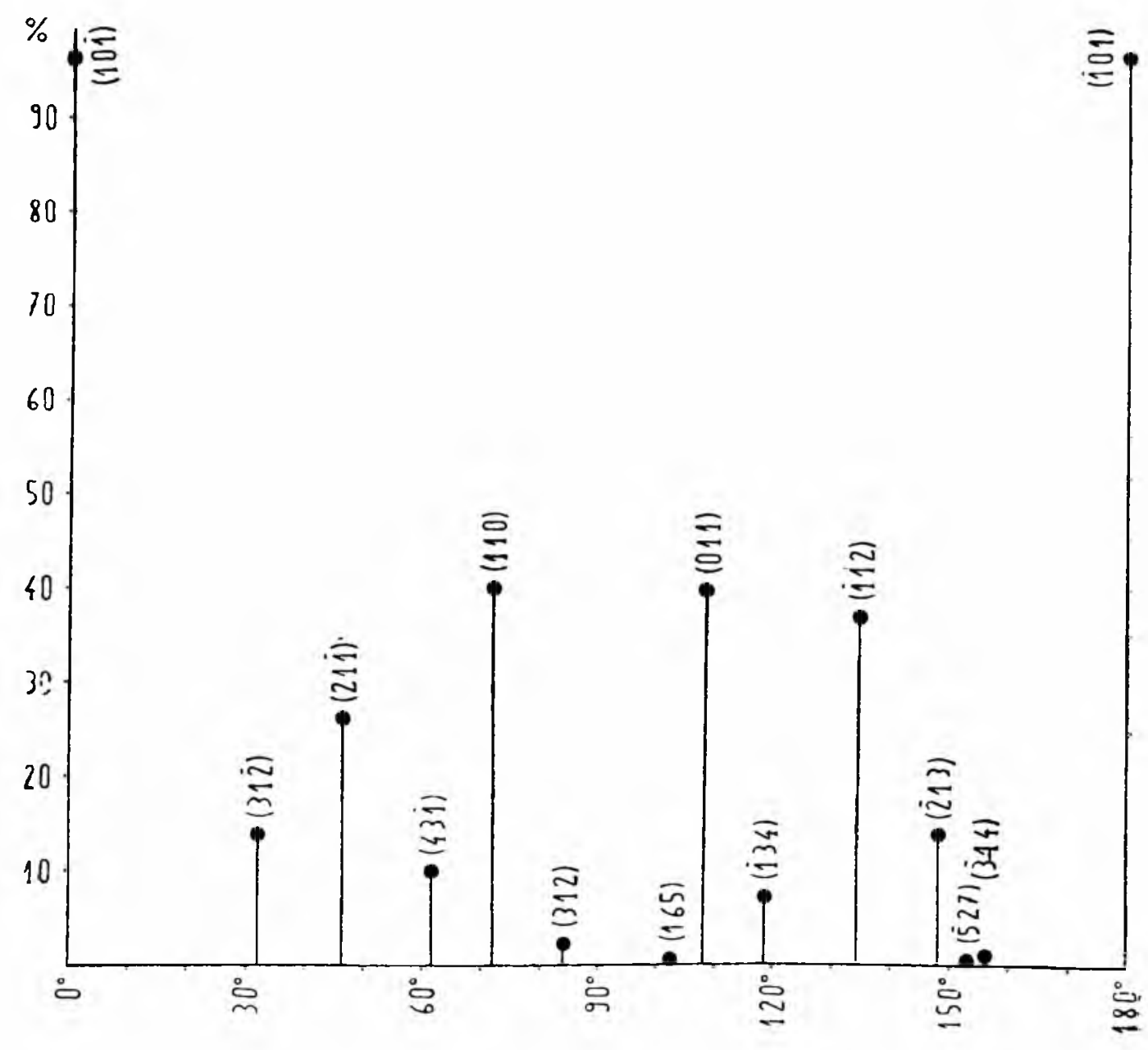

Fig. 10 
T A B E L L A V I

ZONA $[111]^{*}$

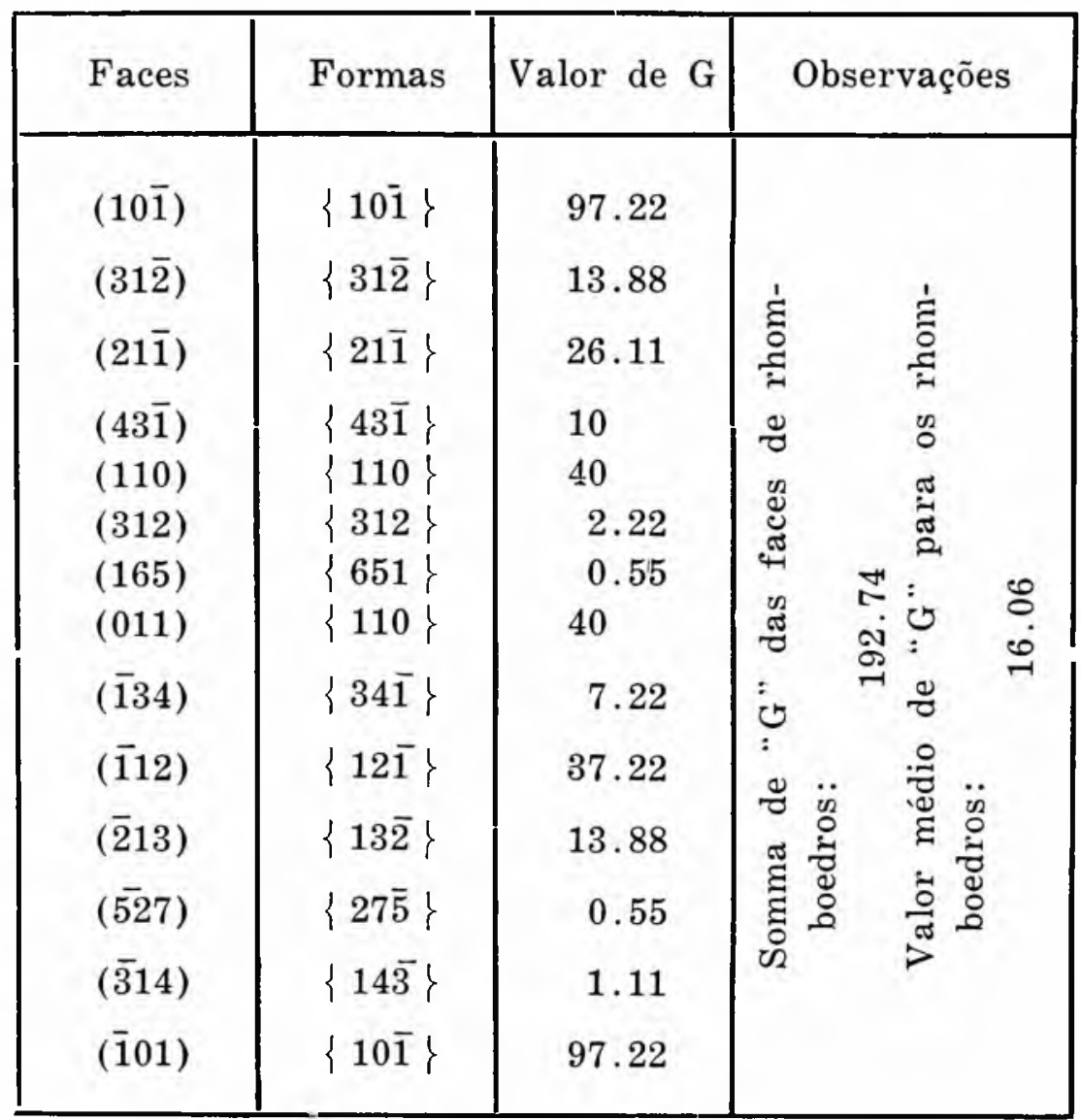

* Da tabella e do graphico apenas constam as faces da metade superior.

Faz-se notar pelo facto de possuir um pequeno numero de faces, mas quasi todas de fórmas a persistencia-desenvolvimento acima da média geral.

Além das zonas fundamental, caracteristicas e especiaes, existem as que exercem funcção muito secundaria e säo uteis poucas vezes para a determinação de fórmas pouco frequentes. Taes zonas não passam por faces dos prismas de $1 .^{\mathrm{a}}$ ou $2 .^{\mathrm{a}}$ ordem e poucas vezes a ellas pertencem os de $3 .^{\mathrm{a}}$. Chamamol-as de complementares e individuaes, seguindo ainda a nomenclatura adoptada por BRASSEUR. (31).

\section{APLICAÇÃO DA LEI DE GOLDSCHMIDT}

Além das conclusões sobre o habitus (assignaladas as fórmas predominantes e zonas caracteristicas) as nossas pesquizas tambem

(31) H. Brasseur - Ob. cit. pg. 193. 
permittiram chegar a uma contribuição experimental para a lei da complicação ennunciada por GoLDSCHMIDT.

Fizemos a combinação dos elementos colhidos no terreno estatistico, procurando relacionar as persistencias-desenvolvimento das fórmas e as importancias das zonas que resultaram das tabellas e graphicos atraz apresentados.

Sendo as fórmas $\{10 \overline{1}\}$ e $\{210\}$, entre os prismas e os rhomboedros, as de mais alta posição na curva (fig. 7) partimos das suas faces (Quadro II) e obtivemos as zonas dos grupos [221] e [100] zonas caracteristicas dos crystaes de S. Miguel de Piracicaba - cujas intersecções representam as faces das fórmas $\{201\},\{100\} \mathrm{e}$ $\{22 \overline{1}\}$, predominantes. Logo de inicio utilisamo-nos tambem do prisma $\{2 \overline{1} \overline{1}\}$ para determinação apenas da zona $\{111\}$ que não nos foi mais necessaria para as outras fórmas constantes do quadro II, todas ellas rhomboedros.

Proseguindo na aplicação da lei encontramos as zonas dos gru-

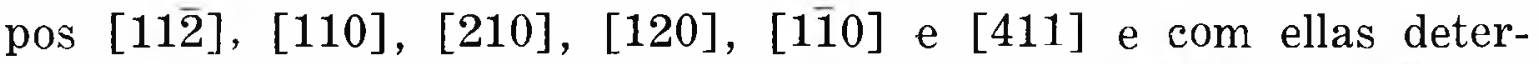
minamos as fórmas $\{110\},\{20 \overline{1}\},\{2 \overline{1} 0\},\{21 \overline{1}\}$ e $\{12 \overline{1}\}$ de grande influencia no habitus. As zonas dos grupos [131] e [111]] decorrem destas e dão, por sua vez, as fórmas $\{411\}$, $\{31 \overline{2}\},\{13 \overline{2}\},\{43 \overline{1}\}$ e $\{34 \overline{1}\}$, as tres primeiras ainda da segunda cathegoria $(G>10)$ e as duas ultimas de persistencia desenvolvimento inferior a 10 , porém muito proximas do limite superior

Podem ser derivadas destas fórmas novos grupos de zonas, [115],

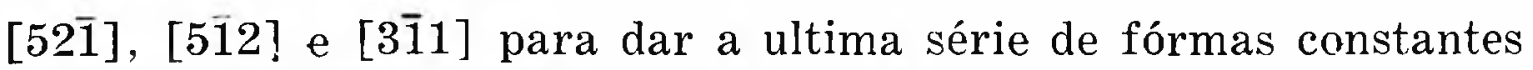
da tabella, $\{3 \overline{1} 1\},\{3 \overline{1} 1\},\{41 \overline{1}\},\{4 \overline{1} 1\}$ e $\{312\}$, todas a persistencia-desenvolvimento inferior a 10 .

$\mathrm{Na}$ complicaç̃o successiva de faces e zonas fizemos intervir, em certas linhas, algumas já encontradas mais acima e que estão por isso assignaladas. Tambem não procuramos determinar todas as faces de uma mesma fórma ou todas as zonas de um mesmo grupo por julgarmos desnecessario complicar inutilmente a tabella. As faces e as zonas correspondentes ás encontradas podem ser incluidas, de maneira facil, sempre por um processo identico.

As rórmas dos ultimos degráos da curva de persistencia desenvolvimento (fig. 7) seriam determinadas com as zonas já inscriptas na tabella ou as complementares e individuaes que nella não figuram. 
A lei da complicação, com é sabido, foi ennunciada theoricamente para crystaes que se desenvolvem em meios de maxima pureza (32) e em condicções ideaes de equilibrio. Não occorrendo isso communmente na natureza é facil prever que a lei não seja verificada totalmente. No nosso caso, por exemplo, deveriam existir certas fórmas, como $\{111\}$ e outras, no cruzamento de zonas caracteristicas e especiaes e que, entretanto, não occorrem, nem mesmo como raras.

Das nossas pesquizas sobre a phenacita de S. Miguel de Piracicaba resulta, porém, que a lei de GoldSCHMIDT é confirmada na sua linha fundamental.

(32) A influencia de pequenas percentagens de impuresas chimicas sobre o habitus dos crystaes foi verificada experimentalmente por H. E. BUCKLEY - (F'urther examples of habit - variation in crystals of potassium permanyanate) - Zeit. f. Krist., 80, 1931, pgs. 238-254. 


\section{SUMMARIUM}

E S. MIGUEL DE PIRACICABA PHENACITAE MORPHOLOGIA ET RATIONES

E S. Miguel de Piracicaba phenacita crystallis plerumque biterminatis apparet decem centimetra amplitudine interdum excedentibus. lucida.

Color lacteus. Exemplaria minora incoloria et eleganter per-

Quas reperimus formas:

$$
\begin{aligned}
& \mathrm{m}\{10 \overline{1}\}, \mathrm{a}\{\overline{21} \overline{1}\}, \mathrm{b}\{3 \overline{1} \overline{2}\} * *, \mathrm{n}\{\overline{514}\}^{*}, \mathrm{n}^{\prime}\{\overline{541}\}^{*} \quad \mathrm{R}\{100\} \text {, } \\
& \mathrm{r}\{411\} \mathrm{R}^{\prime}\{22 \overline{1}\}, \mathrm{r}^{\prime}\{110\}, \mathrm{c}\{11 \overline{1}\}^{* *}, \mathrm{j}\{441\}^{*} \mathrm{p}\{210\}, \mathrm{o}\{31 \overline{1}\} \text {, } \\
& \text { d }\{52 \overline{1}\} * \text {, v }\{11,2, \overline{7}\} * \text { p' }\{201\}, \text { o' }\{\overline{1} \overline{1} 1\}, \text { d' }\{5 \overline{1} 2\} * \text { t }\{312\} * \text {, } \\
& \mathrm{z}\{713\} * \text { ?, x }\{714\} * \text { ?, y }\{534\} \text { ? s }\{20 \overline{1}\}, s^{\prime}\{2 \overline{1} 0\}, e\{21 \overline{1}\} \text {, } \\
& \mathrm{e}^{\prime}\{12 \overline{1}\}, \mathrm{f}\{31 \overline{2}\}, \mathrm{f}^{\prime}\{13 \overline{2}\}^{*}, \mathrm{~g}\{43 \overline{1}\}^{* *}, \mathrm{~g}^{\prime}\{34 \overline{1}\}^{* * *}, \mathrm{k}\{62 \overline{1}\} \text {, } \\
& \mathrm{k}^{\prime}\{6 \overline{1} 2\}^{*}, \mathrm{~h}\{4 \mathbf{1} \overline{\mathbf{1}}\}, \mathrm{h}^{\prime}\{\overline{4} \overline{1} 1\}, \mathrm{l}\{310\}, \mathrm{q}\{14 \overline{3}\}^{*}, \mathrm{u}\{52 \overline{4}\}^{* *} \text {, } \\
& \mathrm{w}\{651\}^{*} ?, \omega\{78 \overline{3}\} * ?, \mathrm{i}\{85 \overline{1}\} *, \alpha\{831\} *, \varepsilon\{8 \overline{12}\} * ?, \eta\{27 \overline{5}\} * \text {, } \\
& \lambda\{10, \overline{7}, \overline{11}\} * \text { ?, } \mathrm{H}\{14,4, \overline{3}\} *, \mathrm{H}_{1}\{14, \overline{3}, 4\}^{*}, \mathrm{~K}\{10, \overline{2}, 3\} *, \mathrm{~K}_{1}\{16,5,3\} *
\end{aligned}
$$

Ex his quattuor et viginti (*) species insueta est ac quinque (**) brasiliensis phenacita.

Constans: $\alpha=108^{\circ} 0^{\prime} 53^{1 / 3}$ "

Habitus: prismaticus ac prismaticus cum complanatione rhomboëdricis formis praevalentibus.

Ex investigatione rationaria de phenacita e S. Miguel de Piracicaba colligitur legem Goldschmidtianam ultimo genere confirmari 